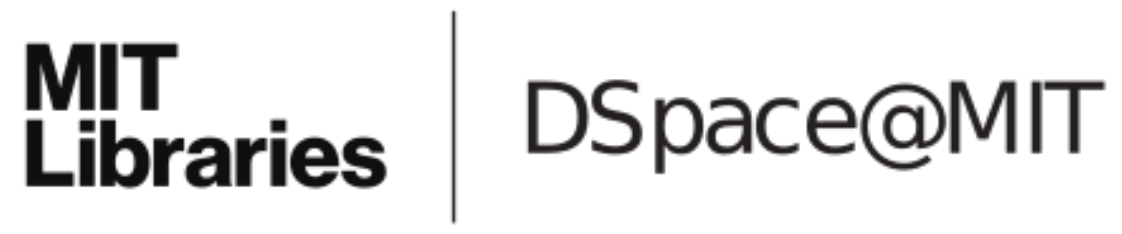

\author{
MIT Open Access Articles
}

\section{Implementation of the Many-Pair Expansion for Systematically Improving Density Functional Calculations of Molecules}

The MIT Faculty has made this article openly available. Please share how this access benefits you. Your story matters.

Citation: Zhu, Tianyu, Piotr de Silva, and Troy Van Voorhis. "Implementation of the ManyPair Expansion for Systematically Improving Density Functional Calculations of Molecules." Journal of Chemical Theory and Computation 15, 2 (February 2019): 1089-101 doi 10.1021/ ACS.JCTC.8B01127 (C2019 Author(s)

As Published: 10.1021/ACS.JCTC.8B01127

Publisher: American Chemical Society (ACS)

Persistent URL: https://hdl.handle.net/1721.1/129453

Version: Author's final manuscript: final author's manuscript post peer review, without publisher's formatting or copy editing

Terms of Use: Article is made available in accordance with the publisher's policy and may be subject to US copyright law. Please refer to the publisher's site for terms of use. 


\title{
Implementation of the Many-Pair Expansion for Systematically Improving Density Functional Calculations of Molecules
}

\author{
Tianyu Zhu, ${ }^{\dagger, \ddagger}$ Piotr de Silva, ${ }^{\dagger}, \boldsymbol{\Phi}$ and Troy Van Voorhis ${ }^{*, \dagger}$ \\ $\dagger$ Department of Chemistry, Massachusetts Institute of Technology, 77 Massachusetts \\ Avenue, Cambridge, Massachusetts 02139, United States \\ $\ddagger$ Present address: Division of Chemistry and Chemical Engineering, California Institute of \\ Technology, Pasadena, California 91125, United States \\ ฯ Present address: Department of Energy Conversion and Storage, Technical University of \\ Denmark, 2800 Kgs. Lyngby, Denmark \\ E-mail: tvan@mit.edu
}

\begin{abstract}
Density functional theory (DFT) is the method of choice for predicting structures and reaction energies of molecular systems. However, it remains a daunting task to systematically improve the accuracy of an approximate density functional. The recentlyproposed many-pair expansion (MPE) [Phys. Rev. B 2016, 93, 201108] is a density functional hierarchy that systematically corrects any deficiencies of an approximate functional to converge to the exact energy, and was shown to give accurate results for lattice models. In this work, we extend MPE to molecular systems and implement it using Gaussian basis sets. The self-attractive Hartree (SAH) decomposition [J. Chem. Theory Comput. 2018, 14, 92-103] is employed to generate localized v-representable pair densities for performing MPE calculations. We demonstrate that MPE at the
\end{abstract}


second order (MPE2) already predicts accurate molecular and reaction energies for a series of small molecules and hydrogen chains, with the EXX functional as its starting point. We also show that MPE correctly describes the symmetric bond breaking in hydrogen rings, indicating its ability to remove strong correlation errors. MPE thus provides a promising framework to systematically improve density functional calculations of molecules.

\section{Introduction}

Density functional theory (DFT) has been the workhorse for quantum mechanical simulations of molecules and solids due to its high accuracy to cost ratio. ${ }^{1-3}$ Although there exists a universal density functional that predicts the exact ground-state electronic properties, ${ }^{4}$ its analytic form is unknown. The Kohn-Sham (KS) framework ${ }^{5}$ is thus employed to perform DFT calculations, in which the exchange-correlation (XC) functional is normally approximated. Despite the great success and popularity of KS-DFT, its application can still suffer from large systematic errors because of the use of approximated XC functionals. ${ }^{6}$ One major reason for systematic failures in density functional approximations is the self-interaction error (SIE), also known as the delocalization error, which originates from the dominating Coulomb

term that pushes electrons apart. ${ }^{7-9}$ The self-interaction error leads to underestimation of the band gaps of materials, the barriers of chemical reactions and the charge transfer excitation energies $^{10,11}$ for commonly-used semi-local density functionals. Another systematic failure for approximate DFT calculations is their inability to describe degenerate or near-degenerate states, which often appear in the breaking of chemical bonds, transition metal systems and strongly correlated materials. ${ }^{12-14}$ Such behavior occurs when the independent-particle picture breaks down, and is attributed to the strong (or static) correlation error. Furthermore, semi-local functionals cannot properly account for long-range interactions because non-local contributions to the electron correlation are missing, ${ }^{15,16}$ putting serious limitations on applicability of DFT to intermolecular interactions. 
Specific functionals and methods have been developed to overcome these systematic errors. For the self-interaction error, the most common solution is to add the exact exchange (EXX) into the XC functional, giving rise to hybrid ${ }^{17}$ and range-separated ${ }^{18}$ functionals. In addition, the Perdew-Zunger self-interaction correction ${ }^{7}$ is designed to remove the spurious one-electron self-interaction in approximate functionals, though its performance is often not satisfactory. ${ }^{19}$ Recently, the localized orbital scaling correction (LOSC) method shows promise for removing the delocalization error by properly treating the fractional electron distribution. ${ }^{20}$ Efforts to correct strong correlation errors include adding a Hubbard-like repulsion term $(\mathrm{DFT}+\mathrm{U}),{ }^{21}$ combining DFT with density matrix renormalization group or multi-configurational self-consistent field calculations, ${ }^{22,23}$ or using the strong-interaction limit of the Hohenberg-Kohn functional. ${ }^{24}$ To correctly account for long-range interactions, particular functionals have been designed by adding an empirical dispersion energy correction term, ${ }^{25-28}$ including a non-local van der Waals correlation functional ${ }^{29-31}$ or using unoccupied Kohn-Sham orbitals. ${ }^{32,33}$ However, none of these methods is capable of curing all problems simultaneously as they are tailored to particular physical conditions. Therefore, the biggest challenge in density functional development is probably to design a scheme to systematically improve the approximate functionals. One such possible DFT hierarchy is represented by Perdew's "Jacob's Ladder". ${ }^{34}$ According to the ingredients (density, density gradient, kinetic energy density, KS orbitals, etc.) utilized in approximate functionals, one puts the functionals onto different rungs of this hierarchy. The higher rungs are expected to give better accuracy as they use more design ingredients, but this is not necessarily true. In practice, one still needs to carefully benchmark the performance of approximate functionals for a particular class of problems before drawing any reliable conclusions from approximate DFT calculations.

To address this problem, we have recently proposed the many-pair expansion (MPE), ${ }^{35}$ which is a hierarchy of density functional approximations that systematically corrects any deficiencies of an approximate functional to finally converge to the true ground-state en- 
ergy. We have shown that MPE gives accurate results for several important lattice models, including 1D/2D Hubbard ${ }^{36}$ and 1D Peierls-Hubbard ${ }^{37}$ models, indicating its capability of correcting strong correlation and self-interaction errors. In addition, we have applied MPE to polyacetylene as well as stacks of ethylene and benzene molecules in the Pariser-Parr-Pople (PPP) lattice model ${ }^{38,39}$ and demonstrated it deals very well with long-range Coulomb interactions. ${ }^{40}$ In previous studies, the usefulness of MPE has only been proved in model Hamiltonians. Therefore, in this work, we extend MPE to real molecules by implementing it using ab initio Hamiltonians in Gaussian basis sets. By employing the recently-developed selfattractive Hartree (SAH) decomposition, ${ }^{41}$ we obtain localized and $v$-representable density fragments, which allows us to perform numerically stable MPE calculations for molecules. We test the performance of MPE for the thermochemistry of a few small molecules and hydrogen chains as well as the symmetric bond breaking of hydrogen rings, and show that low orders of expansion are sufficient to obtain accurate results. Furthermore, we show that this method is numerically convergent for various systems and problems tested, suggesting its potential to serve as a systemically improvable density functional hierarchy for molecular systems.

\section{Theory}

\subsection{Many-Pair Expansion}

We first describe the general formalism of the many-pair expansion (MPE) for spin-compensated systems, using the water molecule as an example, as shown in Figure 1. An extended formalism for spin-uncompensated systems (the many-electron expansion, MEE) can be found in Ref. ${ }^{35}$ Given a $2 N$-electron system, we decompose its total electron density $\rho_{T}(\mathbf{r})$ into a sum of pair densities $\left\{\rho_{i}(\mathbf{r})\right\}$ :

$$
\rho_{T}(\mathbf{r})=\sum_{i=1}^{N} \rho_{i}(\mathbf{r}), \quad \int \rho_{i}(\mathbf{r}) d \mathbf{r}=2 .
$$


Note that by a pair density we mean one-particle density that integrates to two electrons and not a two-particle density $\rho\left(\mathbf{r}, \mathbf{r}^{\prime}\right)$. For the water molecule, one obtains five pair densities from the density decomposition (1-pair terms in Figure 1). We will later stipulate that these pair densities need to be numerically $v$-representable. ${ }^{42}$ We can further construct two-pair, three-pair, ... densities by adding several of the decomposed pair densities (2-pair and 3-pair terms in Figure 1). Then, assume we can compute the energy of any given electron density $\rho(\mathbf{r})$ with an approximate density functional $E_{a}[\rho]$ while we can obtain the exact energy $E_{v}[\rho]$ only for a few electrons at once. Computing the exact energy $E_{v}[\rho]$ is equivalent to solving the full configuration interaction (FCI) problem ${ }^{43,44}$ constrained to give $\rho(\mathbf{r})$ as the ground-state density. This is known as the Lieb construction of density functionals and has been used to compute the adiabatic-connection curves for small molecular systems. ${ }^{45-47}$ The energy correction for $\rho(\mathbf{r})$ can thus be defined as $\Delta E[\rho] \equiv E_{v}[\rho]-E_{a}[\rho]$. We then consider the following hierarchy of approximations to the true energy of this $2 N$-electron system:

$$
\begin{aligned}
E_{0}\left[\rho_{T}\right] & \equiv E_{a}\left[\rho_{T}\right], \\
E_{1}\left[\left\{\rho_{i}\right\}\right] & \equiv E_{0}\left[\rho_{T}\right]+\sum_{i}^{N} \Delta E\left[\rho_{i}\right], \\
E_{2}\left[\left\{\rho_{i}\right\}\right] & \equiv E_{1}\left[\left\{\rho_{i}\right\}\right]+\sum_{i<j}^{N}\left(\Delta E\left[\rho_{i}+\rho_{j}\right]-\Delta E\left[\rho_{i}\right]-\Delta E\left[\rho_{j}\right]\right), \\
E_{3}\left[\left\{\rho_{i}\right\}\right] & \equiv E_{2}\left[\left\{\rho_{i}\right\}\right]+\sum_{i<j<k}^{N}\left(\Delta E\left[\rho_{i}+\rho_{j}+\rho_{k}\right]-\Delta E\left[\rho_{i}+\rho_{j}\right]-\Delta E\left[\rho_{j}+\rho_{k}\right]-\Delta E\left[\rho_{i}+\rho_{k}\right]\right. \\
& \left.+\Delta E\left[\rho_{i}\right]+\Delta E\left[\rho_{j}\right]+\Delta E\left[\rho_{k}\right]\right),
\end{aligned}
$$$$
\text { ... }
$$

In this many-pair expansion, we start from the approximate DFT result $\left(E_{0}\left[\rho_{T}\right]\right)$ and systematically correct its error by applying corrections calculated for a few electrons at a time. As shown in Eq. 2, only $E_{0}\left[\rho_{T}\right]$ is an explicit functional of $\rho_{T}(\mathbf{r})$, while all higher orders are functionals of the pair densities $\left\{\rho_{i}(\mathbf{r})\right\}$. Once the density decomposition (Eq. 1) 


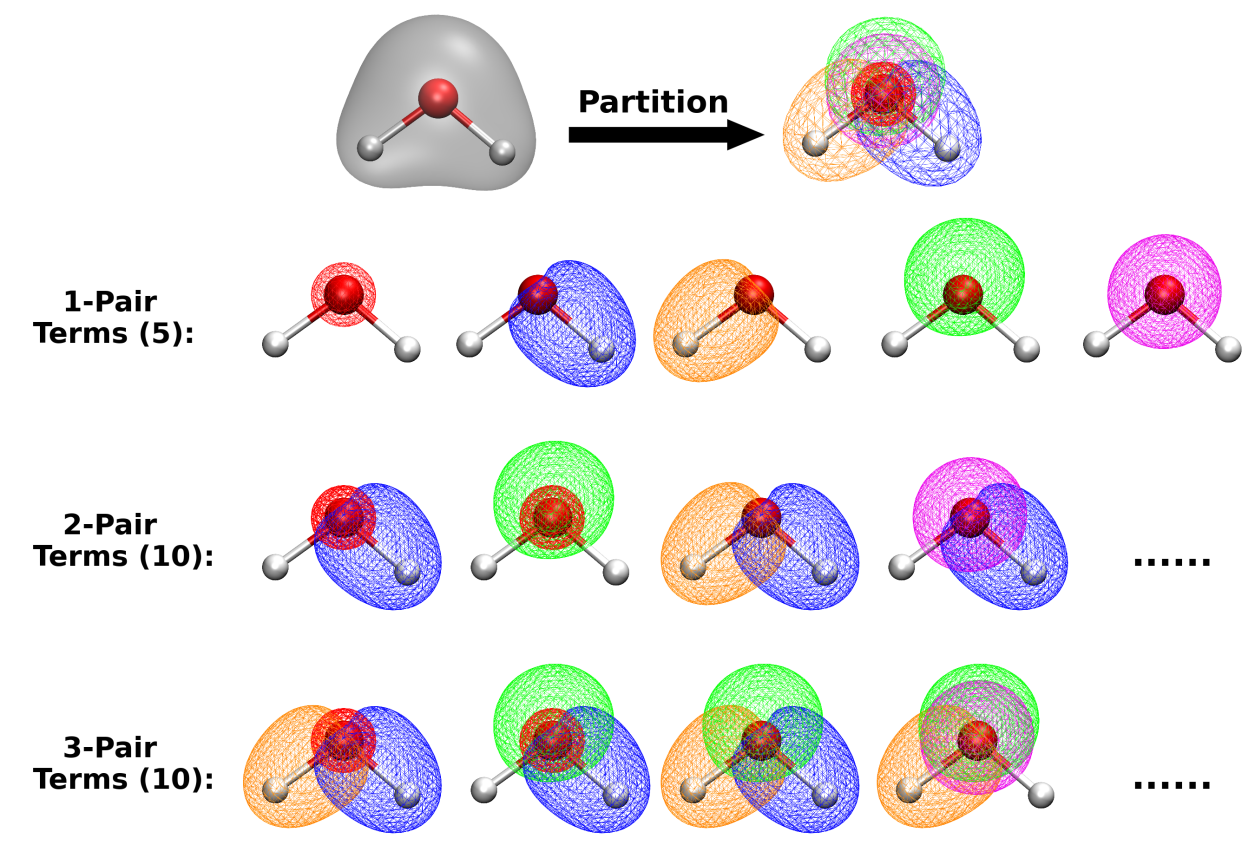

Figure 1: Illustration of MPE using the water molecule as an example.

is prescribed, MPE becomes an implicit functional of the total density $\rho_{T}(\mathbf{r})$. This is similar to orbital-dependent functionals, ${ }^{48}$ which are implicit functionals of the density, through explicit dependence on orbitals defined by Kohn-Sham equations. We also want to point out that MPE is closely related to the many-body expansion for intermolecular interactions, ${ }^{49,50}$ and the method of increments. ${ }^{51} \mathrm{MPE}$ has the important property that $E_{N}\left[\rho_{T}\right]$ gives the exact energy for $2 N$ electrons no matter what approximate functional $E_{a}\left[\rho_{T}\right]$ is chosen. It thus provides a hierarchy of approximations within the context of DFT analogous to the many-body theory for the Green's function ${ }^{52}$ and the coupled-cluster expansion of the wave function. ${ }^{53}$

In order to compute the MPE energies, we need the approximate DFT and exact groundstate energies for various fragment densities $\rho_{q}(\mathbf{r})\left(\rho_{q}(\mathbf{r})\right.$ can be any one-, two-, three-, $\ldots$ pair density). Assuming the approximate density functional is defined within the KohnSham framework, $E_{a}\left[\rho_{q}\right]$ can be obtained via potential inversion techniques. ${ }^{54-56}$ To solve a non-interacting potential inversion problem for a given density $\rho_{q}(\mathbf{r})$, we search for a local 
potential $v_{s}(\mathbf{r})$ such that

$$
\rho\left[v_{s}\right](\mathbf{r})=\rho_{q}(\mathbf{r})
$$

i.e., the input density $\rho_{q}(\mathbf{r})$ is the ground-state density of a non-interacting system in the sought external potential $v_{s}(\mathbf{r})$. The non-interacting Slater determinant $\Phi$ is constructed out of orbitals $\left\{\phi_{k}(\mathbf{r})\right\}$. To find the orbitals $\left\{\phi_{k}(\mathbf{r})\right\}$, we search for the stationary point of the Lagrangian

$$
L_{K S}\left[\left\{\phi_{k}\right\}, v_{s}\right]=\left\langle\Phi\left|-\frac{1}{2} \nabla^{2}\right| \Phi\right\rangle+\int v_{s}(\mathbf{r})\left(\rho(\mathbf{r})-\rho_{q}(\mathbf{r})\right) d \mathbf{r},
$$

where $v_{s}(\mathbf{r})$ is the Lagrange multiplier enforcing the density constraint. For a given $v_{s}(\mathbf{r})$, each KS orbital $\phi_{k}(\mathbf{r})$ satisfies a one-electron Schrödinger equation:

$$
-\frac{1}{2} \nabla^{2} \phi_{k}(\mathbf{r})+v_{s}(\mathbf{r}) \phi_{k}(\mathbf{r})=\epsilon_{k} \phi_{k}(\mathbf{r})
$$

and the density can then be expressed as

$$
\rho\left[v_{s}\right](\mathbf{r})=2 \sum_{k}^{o c c} \phi_{k}(\mathbf{r})^{2} .
$$

Since the potential $v_{s}(\mathbf{r})$ that yields $\rho_{q}(\mathbf{r})$ through Eqs. 5 and 6 is not known, we need to find it numerically to satisfy Eq. 3. We start with an initial guess $v_{s}^{0}(\mathbf{r})$ and use Newton's $\operatorname{method}^{57}$ to find an improved potential

$$
v_{s}^{j+1}(\mathbf{r})=v_{s}^{j}(\mathbf{r})+\int\left(\left.\frac{\delta \rho\left(\mathbf{r}^{\prime}\right)}{\delta v_{s}(\mathbf{r})}\right|_{v_{s}^{j}}\right)^{-1}\left(\rho_{q}\left(\mathbf{r}^{\prime}\right)-\rho\left[v_{s}^{j}\right]\left(\mathbf{r}^{\prime}\right)\right) d \mathbf{r}^{\prime}
$$

where $\delta \rho\left(\mathbf{r}^{\prime}\right) / \delta v_{s}(\mathbf{r})$ denotes the response kernel, which can be computed analytically from the perturbation theory

$$
\frac{\delta \rho\left(\mathbf{r}^{\prime}\right)}{\delta v_{s}(\mathbf{r})}=4 \sum_{i}^{o c c} \sum_{a}^{v i r} \frac{\phi_{i}(\mathbf{r}) \phi_{a}(\mathbf{r}) \phi_{i}\left(\mathbf{r}^{\prime}\right) \phi_{a}\left(\mathbf{r}^{\prime}\right)}{\epsilon_{i}-\epsilon_{a}}
$$


The Newton's search using Eqs. 5-8 is iterated until Eq. 3 is satisfied. Then the DFT energy for $\rho_{q}(\mathbf{r})$ can be computed using the converged KS orbitals: $E_{a}\left[\rho_{q}\right]=E_{a}\left[\left\{\phi_{k}\right\}\right]$.

To obtain the exact energy of the same density $\rho_{q}(\mathbf{r})$, we can apply a similar potential inversion construction except that now we are searching for the exact wave function in a fully interacting system. ${ }^{58}$ The interacting wave function $\Psi$ is a stationary point of the Lagrangian

$$
L_{\text {Exact }}\left[\Psi, v_{e x}\right]=\left\langle\Psi\left|\left[\frac{1}{2} \sum_{k} \hat{\mathbf{p}}_{k}^{2}+\sum_{k<l} \frac{1}{\hat{\mathbf{r}}_{k l}}\right]\right| \Psi\right\rangle+\int v_{e x}(\mathbf{r})\left(\rho(\mathbf{r})-\rho_{q}(\mathbf{r})\right) d \mathbf{r},
$$

where $v_{e x}(\mathbf{r})$ enforces the density constraint. The interacting wave function $\Psi$ is the ground state of the interacting Schrödinger equation

$$
\left[\frac{1}{2} \sum_{i} \hat{\mathbf{p}}_{i}^{2}+\sum_{i<l} \frac{1}{\hat{\mathbf{r}}_{i l}}+v_{e x}(\hat{\mathbf{r}})\right]|\Psi\rangle=E|\Psi\rangle
$$

and the corresponding density is

$$
\rho\left[v_{e x}\right](\mathbf{r})=\langle\Psi|\delta(\hat{\mathbf{r}}-\mathbf{r})| \Psi\rangle
$$

The search for the external potential $v_{e x}(\mathbf{r})$ employs a similar algorithm as in the noninteracting case, except that we use numerical finite differences to compute the response kernel $\delta \rho\left(\mathbf{r}^{\prime}\right) / \delta v_{e x}(\mathbf{r})$. Once the converged potential $v_{e x}(\mathbf{r})$ is found, the exact energy can be obtained as the expectation value of the interacting Hamiltonian $E_{v}\left[\rho_{q}\right]=\langle\Psi|\hat{\mathbf{H}}| \Psi\rangle$.

From the process of computing $E_{a}\left[\rho_{q}\right]$ and $E_{\text {ex }}\left[\rho_{q}\right]$, it is clear that $\rho_{q}(\mathbf{r})$ must be the ground-state density of some potential so that we can find its corresponding wave function and energy. In other words, the fragment density $\rho_{q}(\mathbf{r})$ must be non-interacting and interacting $v$-representable, which needs to be enforced by the density decomposition in Eq. 1. Although the sufficient conditions for $v$-representability are not known, ${ }^{59}$ this is unlikely to be a problem in practice provided that pair densities are sufficiently smooth. Note that even non- $v$-representable densities can be approached arbitrarily close with smooth potentials ${ }^{60,61}$ 
and that within the finite basis set approximation even node-containing densities can be effectively $v$-representable. ${ }^{62}$ Thus, as long as the density is fairly well-behaved (smooth and without prominent nodes) one expects it to be approximately $v$-representable in a suitably large basis. In previous MPE studies on lattice models, ${ }^{35,40}$ the $v$-representability condition is fulfilled by requiring the pair densities to be non-negative on lattice sites.

In this paper, we only discuss the theory and applications of MPE for closed-shell systems. However, it should be pointed out a more general formalism, many-electron expansion (MEE), ${ }^{35}$ can be implemented to deal with open-shell systems. Two possible implementations may be explored in the future. First, a straightforward way is to partition the spin-up and spin-down total densities separately to obtain one-electron fragment densities. One can then perform energy corrections for every one-electron density, every pair of one-electron densities and so on, to form a series of MEE corrections. Second, another possible solution is to adopt a restricted open-shell formalism. For example, for $\mathrm{H}_{2} \mathrm{O}^{-}$, the total density may be decomposed into 5 pair densities and 1 one-electron density, after which MEE energy corrections can be carried out based on these fragment densities.

The MPE hierarchy can also be seen as a method to systematically remove the selfinteraction error in approximate density functionals. The many-electron expansion (which uses one-electron density as the smallest fragment) at the first order (MEE1) calculated with KS orbital densities is equivalent to Perdew-Zunger self-interaction correction. ${ }^{7}$ However, such densities are not admissible in MEE (MPE) because they exhibit pronounced nodal surfaces and are not $v$-representable. Thus, MEE1 can be viewed as the PZ-SIC calculated with smooth $v$-representable densities. Whereas PZ-SIC only corrects one-electron selfinteraction errors, MPE (MEE) is capable of removing many-electron self-interaction ${ }^{63,64}$ to arbitrary order. In many situations self-interaction mimics correlation in DFT, so that removing self-interaction without adding correlation can make the results worse. ${ }^{19,65}$ At each order, MPE compensates for the excluded many-electron self-interaction by including a corresponding degree of many-electron correlation. Thus, MPE is in some sense balanced, 
even at low orders.

\subsection{Self-Attractive Hartree Decomposition}

The $v$-representability requirement for pair densities makes the implementation of molecular MPE difficult, because there is no easy way to partition the molecular density into sufficiently smooth fragments in finite Gaussian basis sets. In addition, we also require the pair densities to be localized to accelerate the convergence of MPE series so that low orders of expansion are enough for accurate results. This localization requirement is similar to employing localized molecular orbitals (LMOs) ${ }^{66}$ in the method of increments. ${ }^{67}$ Unfortunately, pair densities constructed out of LMOs cannot meet the $v$-representability criterion in MPE due to the existence of nodes. Another straightforward idea may be partitioning the density of a $2 N-$ electron system equally into $N$ pair densities. In that case, the pair densities are satisfactorily smooth but highly delocalized, leading to poor convergence of MPE expansions.

To tackle this problem, we recently developed a new density partitioning method - selfattractive Hartree (SAH) decomposition, ${ }^{41}$ which is capable of generating smooth and localized pair densities to be used in molecular MPE. Here, we recapitulate the formalism of the SAH decomposition. We start by minimizing a regularized Lagrangian

$$
\begin{aligned}
L\left[\left\{\phi_{i}\right\}, \mu(\mathbf{r}),\left\{\epsilon_{i}\right\}\right]= & -\alpha \cdot \frac{1}{2} \sum_{i}^{N} \iint \frac{\rho_{i}(\mathbf{r}) \rho_{i}\left(\mathbf{r}^{\prime}\right)}{\left|\mathbf{r}-\mathbf{r}^{\prime}\right|} d \mathbf{r} d \mathbf{r}^{\prime}+\sum_{i}^{N} \int\left|\nabla \phi_{i}(\mathbf{r})\right|^{2} d \mathbf{r} \\
& +\int \mu(\mathbf{r})\left(\sum_{i}^{N} \rho_{i}(\mathbf{r})-\rho_{T}(\mathbf{r})\right) d \mathbf{r}-\sum_{i}^{N} \epsilon_{i}\left(\int \rho_{i}(\mathbf{r}) d \mathbf{r}-2\right),
\end{aligned}
$$

where the pair densities are expressed in terms of non-orthogonal auxiliary orbitals

$$
\rho_{i}(\mathbf{r})=2\left|\phi_{i}(\mathbf{r})\right|^{2}
$$

The first term in Eq. 12 is the sum of self-attraction energies of pair densities, which is just negative of the self-repulsion. Minimizing this term is similar to the Edmiston-Reudenberg 
(ER) localization, ${ }^{68}$ but with non-orthogonal orbitals. Meanwhile, the second term in Eq. 12 is the sum of the kinetic energies of pair densities, which penalizes any possible sharp features in pair densities. The third and fourth terms in Eq. 12 are just two density constraints in Eq. 1, where $\mu(\mathbf{r})$ and $\left\{\epsilon_{i}\right\}$ are Lagrangian multipliers. Because simultaneous minimization of the self-attraction and kinetic energy are conflicting requirements, minimization of their sum would give solutions that are regularized compared to pure localization solutions. We further add a parameter $\alpha(>0)$ in Eq. 12 that scales the self-attraction energy to control the level of regularization.

Solving for stationary points of the Lagrangian in Eq. 12 gives the following set of $N$ equations for $N$ pair densities:

$$
\left[-\frac{1}{2} \nabla^{2}-2 \alpha \int \frac{\phi_{i}\left(\mathbf{r}^{\prime}\right)^{2}}{\left|\mathbf{r}-\mathbf{r}^{\prime}\right|} d \mathbf{r}^{\prime}+\mu(\mathbf{r})\right] \phi_{i}(\mathbf{r})=\epsilon_{i} \phi_{i}(\mathbf{r})
$$

Eq. 14 has the similar form to the Hartree equation. ${ }^{69}$ Instead of a repulsive potential in the

Hartree equation, we have the self-attractive potential $-2 \alpha \int \frac{\phi_{i}\left(\mathbf{r}^{\prime}\right)^{2}}{\left|\mathbf{r}-\mathbf{r}^{\prime}\right|} d \mathbf{r}^{\prime}$. Therefore, Eq. 14 can be interpreted as describing self-attracting electrons in a common external field $\mu(\mathbf{r})$, which constrains all pair densities to add up to the total density. Thus, this new density partitioning method is named the self-attractive Hartree (SAH) decomposition.

Meanwhile, Eq. 14 has another feature that each $\phi_{i}(\mathbf{r})$ is a ground-state solution of a Kohn-Sham (KS) equation, meaning that each pair density is non-interacting $v$-representable by design. Such $v$-representability feature makes the SAH pair densities the perfect candidate for implementing MPE for molecular systems.

\subsection{Implementation of MPE for Molecules}

To implement the SAH decomposition using standard linear algebra techniques, it is necessary to represent the potential $\mu(\mathbf{r})$ in Eq. 14 in some convenient basis. Here, we introduce an auxiliary basis set $\left\{\chi_{P}(\mathbf{r})\right\}$ composed of Gaussian-type atomic orbitals. ${ }^{70,71}$ Then, the ex- 
ternal potential $\mu(\mathbf{r})$ can be expanded using Coulomb potentials of functions in the auxiliary basis set: ${ }^{72}$

$$
\mu(\mathbf{r})=v_{n u c}(\mathbf{r})+\sum_{t} b_{t} \tilde{\chi}_{t}(\mathbf{r})=v_{n u c}(\mathbf{r})+\sum_{t} b_{t} \int \frac{\chi_{t}\left(\mathbf{r}^{\prime}\right)}{\left|\mathbf{r}-\mathbf{r}^{\prime}\right|} d \mathbf{r}^{\prime},
$$

where $v_{\text {nuc }}(\mathbf{r})$ is the nuclear potential of the system and $\left\{b_{t}\right\}$ are the coefficients for the potential basis functions. The same basis set can be used to represent the self-attraction potential in Eq. 14 by expanding pair densities in the auxiliary basis set

$$
\rho_{i}(\mathbf{r})=2\left|\phi_{i}(\mathbf{r})\right|^{2} \approx 2 \sum_{P} d_{P}^{i} \chi_{P}(\mathbf{r})
$$

with density fitting coefficients ${ }^{73,74}$

$$
d_{P}^{i}=\sum_{Q}(i i \mid Q)(Q \mid P)^{-1}
$$

where

$$
\begin{gathered}
(i i \mid Q)=\iint \frac{\phi_{i}(\mathbf{r}) \phi_{i}(\mathbf{r}) \chi_{Q}\left(\mathbf{r}^{\prime}\right)}{\left|\mathbf{r}-\mathbf{r}^{\prime}\right|} d \mathbf{r} d \mathbf{r}^{\prime} \\
(Q \mid P)=\iint \frac{\chi_{Q}(\mathbf{r}) \chi_{P}\left(\mathbf{r}^{\prime}\right)}{\left|\mathbf{r}-\mathbf{r}^{\prime}\right|} d \mathbf{r} d \mathbf{r}^{\prime}
\end{gathered}
$$

By inserting Eq. 16 and Eq. 15 into Eq. 14, we obtain the final working equation for the SAH decomposition:

$$
\left[-\frac{1}{2} \nabla^{2}+v_{n u c}(\mathbf{r})-2 \alpha \sum_{P} d_{P}^{i} \widetilde{\chi}_{P}(\mathbf{r})+\sum_{t} b_{t} \widetilde{\chi}_{t}(\mathbf{r})\right] \phi_{i}(\mathbf{r})=\epsilon_{i} \phi_{i}(\mathbf{r})
$$

We use a constrained SCF algorithm composed of an outer loop and an inner loop to solve Eq. 20 under the constraints in Eq. 1. In the outer loop, we perform the normal SCF procedure for each pair density to update $\phi_{i}(\mathbf{r})$ (and $\left\{d_{P}^{i}\right\}$ according to Eq. 17) iteratively. Note the constraint $\int \rho_{i}(\mathbf{r}) d \mathbf{r}=2$ is automatically fulfilled by occupying two electrons in the lowest orbital of each $\phi_{i}(\mathbf{r})$. In the inner loop, $\left\{b_{t}\right\}$ is updated for all $N$ SCF equations 
through the $\mathrm{Wu}$-Yang potential inversion technique ${ }^{55,75}$ to satisfy $\sum_{i}^{N} \rho_{i}(\mathbf{r})=\rho_{T}(\mathbf{r})$. For the detailed algorithm, the readers are referred to Ref. ${ }^{41}$

As discussed in Ref., ${ }^{41}$ the quality of SAH pair densities is affected by the choice of the parameter $\alpha$. A small $\alpha$ value results in pair densities that are not localized enough, while a large $\alpha$ value may give rise to sharp changes and nodes in pair densities. Note that although the SAH pair densities are ground-state solutions of Kohn-Sham equations, they can still possess nodes due to the use of finite basis sets. Meanwhile, when the $\alpha$ value is too large, the potential inversion procedures in MPE may not converge. Therefore, we want to choose the maximum $\alpha$ value that still maintains smoothness in pair densities. In this work, we choose $\alpha=4$ for all tested molecules and show the dependence of MPE energies on the choice of $\alpha$ is weak in a later section.

As can be seen in Figure 1, the SAH pair densities for the water molecule are localized and smooth. We then construct relevant fragment densities $\rho_{q}(\mathbf{r})$ and compute the DFT energy $E_{a}\left[\rho_{q}\right]$ as described in Eqs. 3-8, using Wu-Yang potential inversion algorithm. Similar to Eq. 15, we expand the Lagrange multiplier $v_{s}(\mathbf{r})$ in Eq. 4 using Coulomb potentials of functions in the auxiliary basis set $\left\{\chi_{P}(\mathbf{r})\right\}$ :

$$
v_{s}(\mathbf{r})=v_{n u c}(\mathbf{r})+\sum_{P} c_{P} \widetilde{\chi}_{P}(\mathbf{r})=v_{n u c}(\mathbf{r})+\sum_{P} c_{P} \int \frac{\chi_{P}\left(\mathbf{r}^{\prime}\right)}{\left|\mathbf{r}-\mathbf{r}^{\prime}\right|} d \mathbf{r}^{\prime},
$$

where $\left\{c_{P}\right\}$ are the coefficients for the potential basis functions. Then Eq. 4 becomes

$$
L_{K S}\left[\left\{\phi_{k}\right\},\left\{c_{P}\right\}\right]=\left\langle\Phi\left|-\frac{1}{2} \nabla^{2}\right| \Phi\right\rangle+\int\left(v_{n u c}(\mathbf{r})+\sum_{P} c_{P} \tilde{\chi}_{P}(\mathbf{r})\right)\left(\rho(\mathbf{r})-\rho_{q}(\mathbf{r})\right) d \mathbf{r}
$$

and each KS orbital $\phi_{k}(\mathbf{r})$ satisfies the equation

$$
\left(-\frac{1}{2} \nabla^{2}+v_{n u c}(\mathbf{r})+\sum_{P} c_{P} \tilde{\chi}_{P}(\mathbf{r})\right) \phi_{k}(\mathbf{r})=\epsilon_{k} \phi_{k}(\mathbf{r})
$$

The density $\rho\left[v_{s}\right](\mathbf{r})$ can be computed using Eq. 6. To optimize the Lagrangian in Eq. 22, 
we analytically compute the gradient of $L_{K S}$ with respect to coefficients $\left\{c_{P}\right\}$

$$
\frac{\partial L_{K S}}{\partial c_{P}}=\int \tilde{\chi}_{P}(\mathbf{r})\left(\rho\left[v_{s}\right](\mathbf{r})-\rho_{q}(\mathbf{r})\right) d \mathbf{r}
$$

and the Hessian

$$
\begin{aligned}
\frac{\partial^{2} L_{K S}}{\partial c_{P} \partial c_{Q}} & =\iint \tilde{\chi}_{P}(\mathbf{r}) \cdot 4 \sum_{i}^{o c c} \sum_{a}^{v i r} \frac{\phi_{i}(\mathbf{r}) \phi_{a}(\mathbf{r}) \phi_{i}\left(\mathbf{r}^{\prime}\right) \phi_{a}\left(\mathbf{r}^{\prime}\right)}{\epsilon_{i}-\epsilon_{a}} \cdot \widetilde{\chi}_{Q}\left(\mathbf{r}^{\prime}\right) d \mathbf{r} d \mathbf{r}^{\prime} \\
& =4 \sum_{i}^{o c c} \sum_{a}^{v i r} \frac{\left(\phi_{i} \phi_{a} \mid \chi_{P}\right)\left(\phi_{i} \phi_{a} \mid \chi_{Q}\right)}{\epsilon_{i}-\epsilon_{a}}
\end{aligned}
$$

Note that the magnitude of the gradient in Eq. 24 is an indicator for the density matching. With analytic gradient and Hessian, we can use a standard Newton's method to find the optimized potential expansion coefficients $\left\{c_{P}\right\}$. Once the converged KS orbitals $\left\{\phi_{k}(\mathbf{r})\right\}$ are found, the DFT energy for $\rho_{q}(\mathbf{r})$ can be computed: $E_{a}\left[\rho_{q}\right]=E_{a}\left[\left\{\phi_{k}\right\}\right]$.

To obtain the exact energy of the same fragment density $\rho_{q}(\mathbf{r})$, we apply an interacting Wu-Yang potential inversion algorithm. We first expand the potential $v_{\text {ex }}(\mathbf{r})$ in Eq. 9:

$$
v_{e x}(\mathbf{r})=v_{n u c}(\mathbf{r})+\sum_{P} f_{P} \widetilde{\chi}_{P}(\mathbf{r})=v_{n u c}(\mathbf{r})+\sum_{P} f_{P} \int \frac{\chi_{P}\left(\mathbf{r}^{\prime}\right)}{\left|\mathbf{r}-\mathbf{r}^{\prime}\right|} d \mathbf{r}^{\prime}
$$

Then Eq. 9 becomes

$$
\begin{aligned}
L_{\text {Exact }}\left[\Psi,\left\{f_{P}\right\}\right]= & \left\langle\Psi\left|\left[\frac{1}{2} \sum_{k} \hat{\mathbf{p}}_{k}^{2}+\sum_{k<l} \frac{1}{\hat{\mathbf{r}}_{k l}}\right]\right| \Psi\right\rangle \\
& +\int\left(v_{\text {nuc }}(\mathbf{r})+\sum_{P} f_{P} \widetilde{\chi}_{P}(\mathbf{r})\right)\left(\rho(\mathbf{r})-\rho_{q}(\mathbf{r})\right) d \mathbf{r} .
\end{aligned}
$$

and $\Psi$ is the ground state of the interacting Schrödinger equation

$$
\left[\frac{1}{2} \sum_{i} \hat{\mathbf{p}}_{i}^{2}+\sum_{i<l} \frac{1}{\hat{\mathbf{r}}_{i l}}+v_{n u c}(\hat{\mathbf{r}})+\sum_{P} f_{P} \widetilde{\chi}_{P}(\hat{\mathbf{r}})\right]|\Psi\rangle=E|\Psi\rangle
$$

The density $\rho\left[v_{e x}\right](\mathbf{r})$ can be computed using Eq. 11. The optimization of the Lagrangian in 
Eq. 27 can also be performed using a standard Newton's method, as in the non-interacting case. The analytic gradient can be computed as

$$
\frac{\partial L_{\text {Exact }}}{\partial f_{P}}=\int \tilde{\chi}_{P}(\mathbf{r})\left(\rho\left[v_{e x}\right](\mathbf{r})-\rho_{q}(\mathbf{r})\right) d \mathbf{r} .
$$

While the Hessian could be computed analytically via linear response, for now it is computed using the finite difference method. The converged interacting wave function $\Psi$ can then be used to compute the exact energy for the fragment density $\rho_{q}(\mathbf{r}): E_{v}\left[\rho_{q}\right]=\langle\Psi|\hat{\mathbf{H}}| \Psi\rangle$. Note that although there is no guarantee that the SAH pair densities are also interacting $v$ representable, our numerical tests show that one can usually find the corresponding potential $v_{e x}(\mathbf{r})$ in the interacting potential inversion as long as the pair densities are non-interacting $v$-representable in the given basis set.

With the approximate DFT and exact energies for fragment densities, the MPE energies can be finally computed according to Eq. 2 at the desired levels. In this study, due to the high computational cost of interacting potential inversion calculations, we perform MPE calculations up to the second order (MPE2), which means we only correct the DFT energies for one-pair and two-pair densities.

By comparing Eq. 20 and Eq. 23, one may realize that the potential $v_{s}(\mathbf{r})$ in the noninteracting potential inversion for one-pair densities are already pre-defined by the SAH decomposition:

$$
v_{s}(\mathbf{r})=v_{n u c}(\mathbf{r})+\sum_{P} c_{P} \tilde{\chi}_{P}(\mathbf{r})=v_{n u c}(\mathbf{r})-2 \alpha \sum_{P} d_{P}^{i} \widetilde{\chi}_{P}(\mathbf{r})+\sum_{t} b_{t} \widetilde{\chi}_{t}(\mathbf{r}) .
$$

Thus, the expansion coefficients for one-pair densities $\left\{c_{P}\right\}$ can be directly obtained from

$$
c_{P}=-2 \alpha \cdot d_{P}^{i}+b_{P}
$$

without doing the potential inversion procedure in Eqs. 22-25. 


\subsection{Energy Estimation for Non-v-representable Densities}

For fragment densities larger than one-pair densities, although they are very likely to be $v$ representable due to their smooth feature, they are not guaranteed to be $v$-representable in the basis we have chosen. Thus, it may still be difficult to find the corresponding converged potentials for these densities in the potential inversion processes. Without the converged potentials $v_{s}(\mathbf{r})$ and $v_{e x}(\mathbf{r})$, the DFT and FCI energies for the fragment density $E_{a}\left[\rho_{q}\right]$ and $E_{v}\left[\rho_{q}\right]$ cannot be accurately computed. For example, in the water molecule (Figure 1), the two-pair density that comes from adding two lone-pair densities is not $v$-representable in the chosen basis set.

In this section, we describe a numerical method to reduce the energy errors for twopair densities caused by non- $v$-representability. For simplicity, assume such two-pair density comes from adding the first and second pair densities $\rho_{q}(\mathbf{r})=\rho_{1+2}(\mathbf{r})=\rho_{1}(\mathbf{r})+\rho_{2}(\mathbf{r})$. We need to estimate the contribution to the energy correction from this non- $v$-representable twopair density in Eq. 2: $E^{\text {corr }}\left[\rho_{1+2}\right]=\Delta E\left[\rho_{1+2}\right]-\Delta E\left[\rho_{1}\right]-\Delta E\left[\rho_{2}\right]$, where only $\Delta E\left[\rho_{1}\right]$ and $\Delta E\left[\rho_{2}\right]$ can be accurately computed. A straightforward way is to approximately compute the energy correction term $\Delta E\left[\rho_{1+2}\right]$. Although the converged potential for non-interacting potential inversion $v_{s}(\mathbf{r})$ cannot be found, we can instead search for the potential $\widetilde{v}_{s}(\mathbf{r})$ that gives the most closely approximate density $\widetilde{\rho}_{1+2}(\mathbf{r})$, which is the potential that gives the smallest gradient (Eq. 24) norm in the optimization of the Lagrangian in Eq. 22. Using the approximate potential $\widetilde{v}_{s}(\mathbf{r})$, we can estimate the DFT energy for $\rho_{1+2}(\mathbf{r}): E_{a}\left[\rho_{1+2}\right] \approx$ $E_{a}\left[\widetilde{\rho}_{1+2}\right]$. An interacting potential inversion can then be performed exactly for the same approximate density $\widetilde{\rho}_{1+2}(\mathbf{r})$ to obtain its FCI energy $E_{v}\left[\widetilde{\rho}_{1+2}\right]$. Since $\widetilde{\rho}_{1+2}(\mathbf{r})$ is a noninteracting $v$-representable density, we find in practice the convergence of its interacting

potential inversion is normally good. The estimation to $E^{\text {corr }}\left[\rho_{1+2}\right]$ can thus be calculated 
as:

$$
\begin{aligned}
E^{c o r r}\left[\rho_{1+2}\right] & =\Delta E\left[\rho_{1+2}\right]-\Delta E\left[\rho_{1}\right]-\Delta E\left[\rho_{2}\right] \approx \Delta E\left[\widetilde{\rho}_{1+2}\right]-\Delta E\left[\rho_{1}\right]-\Delta E\left[\rho_{2}\right] \\
& \approx E_{v}\left[\widetilde{\rho}_{1+2}\right]-E_{a}\left[\widetilde{\rho}_{1+2}\right]-\Delta E\left[\rho_{1}\right]-\Delta E\left[\rho_{2}\right] .
\end{aligned}
$$

Nevertheless, the approximation in Eq. 32 may still lead to large energy errors when the density error $\|\mathbf{W}\|$ is large, where

$$
W_{p}=\int \widetilde{\chi}_{P}(\mathbf{r})\left(\widetilde{\rho}_{1+2}(\mathbf{r})-\rho_{1+2}(\mathbf{r})\right) d \mathbf{r}
$$

because $\widetilde{\rho}_{1+2}(\mathbf{r}) \neq \rho_{1}(\mathbf{r})+\rho_{2}(\mathbf{r})$. To solve this problem, we introduce a "top-down" correction scheme. Once we get the approximate two-pair density $\widetilde{\rho}_{1+2}(\mathbf{r})$, we use this density as the input to perform a new SAH decomposition to generate two new one-pair densities:

$$
\widetilde{\rho}_{1+2}(\mathbf{r}) \stackrel{\mathrm{SAH}}{\longrightarrow} \widetilde{\rho}_{1}(\mathbf{r})+\widetilde{\rho}_{2}(\mathbf{r})
$$

where $\widetilde{\rho}_{1}(\mathbf{r})$ and $\widetilde{\rho}_{2}(\mathbf{r})$ resemble the original one-pair densities $\rho_{1}(\mathbf{r})$ and $\rho_{2}(\mathbf{r})$. In this way, we rebuild the one-pair densities whose sum equals to $\widetilde{\rho}_{1+2}(\mathbf{r})$ and then estimate $E^{\text {corr }}\left[\rho_{1+2}\right]$ using:

$$
\begin{aligned}
E^{\operatorname{corr}}\left[\rho_{1+2}\right] & =\Delta E\left[\rho_{1+2}\right]-\Delta E\left[\rho_{1}\right]-\Delta E\left[\rho_{2}\right] \approx \Delta E\left[\widetilde{\rho}_{1+2}\right]-\Delta E\left[\widetilde{\rho}_{1}\right]-\Delta E\left[\widetilde{\rho}_{2}\right] \\
& \approx E_{v}\left[\widetilde{\rho}_{1+2}\right]-E_{a}\left[\widetilde{\rho}_{1+2}\right]-\left(E_{v}\left[\widetilde{\rho}_{1}\right]-E_{a}\left[\widetilde{\rho}_{1}\right]\right)-\left(E_{v}\left[\widetilde{\rho}_{2}\right]-E_{a}\left[\widetilde{\rho}_{2}\right]\right) .
\end{aligned}
$$

Here, we compute the DFT and FCI energies for new one-pair densities $\widetilde{\rho}_{1}(\mathbf{r})$ and $\widetilde{\rho}_{2}(\mathbf{r})$, and use those energies to estimate $E^{\operatorname{corr}}\left[\rho_{1+2}\right]$. This is a better cancellation of error compared to Eq. 32 due to the density match $\widetilde{\rho}_{1+2}(\mathbf{r})=\widetilde{\rho}_{1}(\mathbf{r})+\widetilde{\rho}_{2}(\mathbf{r})$.

We apply the top-down correction scheme described in Eq. 35 to the two-pair densities that are non- $v$-representable in the given basis set. For the cases presented in this work, we 
have verified that such correction leads to an estimated error of $<1 \mathrm{kcal} / \mathrm{mol}$ in the energies (See SI for more information), so the basis set incompatibility is not a significant limitation to the method.

\section{Computational Details}

The ground state geometries of tested small molecules are optimized using the coupledcluster singles and doubles (CCSD) method $^{76}$ in the cc-pVTZ basis set ${ }^{77}$ using Q-Chem 4.2 software package. ${ }^{78}$ For SAH decomposition and MPE calculations, two basis sets are used for tests: (a) uncontracted cc-pVDZ, ${ }^{77}$ abbreviated as uDZ; (b) uncontracted cc-pVTZ for nonhydrogen atoms and uncontracted cc-pVDZ for hydrogen atoms, abbreviated as uTZ. The input electron density for all molecules is obtained through Hartree-Fock (HF) calculations in $\mathrm{uDZ}$ and $\mathrm{uTZ}$ basis sets. In this study, we only use the exact exchange (EXX) functional as our approximate density functional, and perform all MPE calculations starting from EXX results. For closed-shell systems, the EXX energy is

$$
E_{x}^{E X X}=-\sum_{i}^{o c c} \sum_{j}^{o c c} \iint \frac{\phi_{i}(\mathbf{r}) \phi_{j}(\mathbf{r}) \phi_{i}\left(\mathbf{r}^{\prime}\right) \phi_{j}\left(\mathbf{r}^{\prime}\right)}{\left|\mathbf{r}-\mathbf{r}^{\prime}\right|} d \mathbf{r} d \mathbf{r}^{\prime}
$$

where $\left\{\phi_{i}(\mathbf{r})\right\}$ are KS orbitals. We choose EXX as the starting point for MPE because it does not require any grid information, while any pure density functional requires basis function information on the grid. Because we use Gaussian basis functions, all the integrals needed for EXX calculations can be computed analytically and retrieved from a standard electronic structure code (e.g., QChem). Meanwhile, no matter what functional is used, we always need to perform potential inversions to get accurate non-interacting kinetic energies for fragment densities, so using pure density functionals cannot avoid non-interacting potential inversions. Therefore, EXX is a reasonable choice for our first implementation study, and we will implement other starting density functionals in the future work.

Coupled-cluster singles and doubles with perturbative triples $(\operatorname{CCSD}(\mathrm{T}))^{79}$ energies are 
computed using Q-Chem 4.2 package as the reference for thermochemistry results. Density matrix renormalization group (DMRG) ${ }^{80}$ energies are calculated using PySCF package ${ }^{81}$ and BLOCK code ${ }^{82,83}$ as the reference for hydrogen ring bond breaking results.

As known from the literature, the potential inversion and optimized effective potential (OEP) techniques are numerically unstable in finite basis sets. ${ }^{84,85}$ Several solutions exist to avoid ill-conditioning problems in potential inversions. ${ }^{86-89}$ Here, we follow the work of Görling and co-workers ${ }^{86}$ to balance the orbital and auxiliary basis sets. This leads to our use of uncontracted orbital basis sets in this work. Meanwhile, the corresponding auxiliary basis set $\left\{\chi_{P}(\mathbf{r})\right\}$ for expanding potentials is carefully constructed out of the orbital basis set by removing some of the most compact and diffuse functions. The detailed description of balanced orbital and auxiliary basis sets can be found in the Supporting Information. $\mathrm{VMD}^{90}$ and Molden ${ }^{91}$ are used to generate fragment density pictures.

\section{Results and Discussion}

\subsection{Thermochemistry}

We first test the performance of MPE on the thermochemistry of molecules. DFT is known to perform very well for thermochemistry due to the effective description of dynamic correlations, ${ }^{6}$ particularly hybrid and double-hybrid functionals. ${ }^{17,92,93}$ However, even for thermochemistry, approximate functionals cannot achieve the chemical accuracy of $1 \mathrm{kcal} / \mathrm{mol}$ for typical chemical reactions. Thus, systematic improvement of density functional calculations is still needed for the accurate description of thermochemistry.

In all test cases for thermochemistry, we use the Hartree-Fock (HF) density as the input to perform MPE calculations. Due to this approximate total electron density, MPE will not converge to the true exact (FCI) energy. Instead, MPE energies converge to the FCI energy constrained to the given HF density, which equals to inverting the full interacting Schrödinger equation with the density constraint. The HF density is known to be a good 
approximation of electron density for equilibrium molecular structures ${ }^{94}$ so we expect it causes small energy errors. Our results above also show the effect of using HF density is small for the description of thermochemistry.

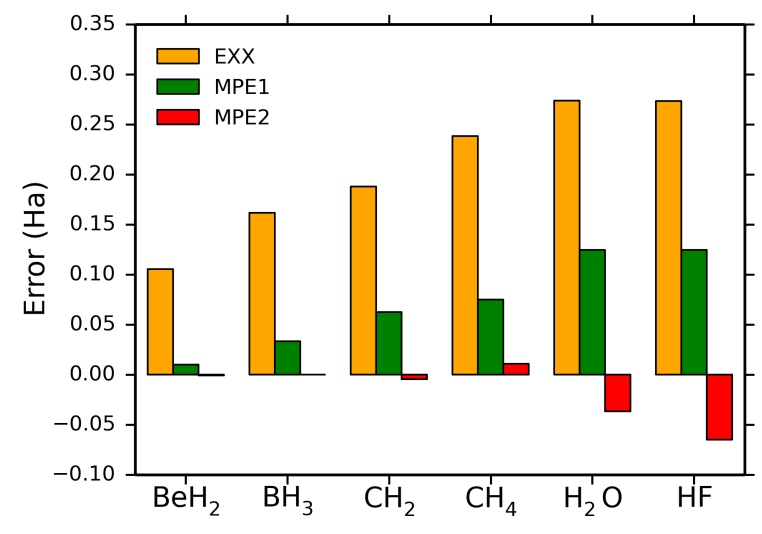

(a)

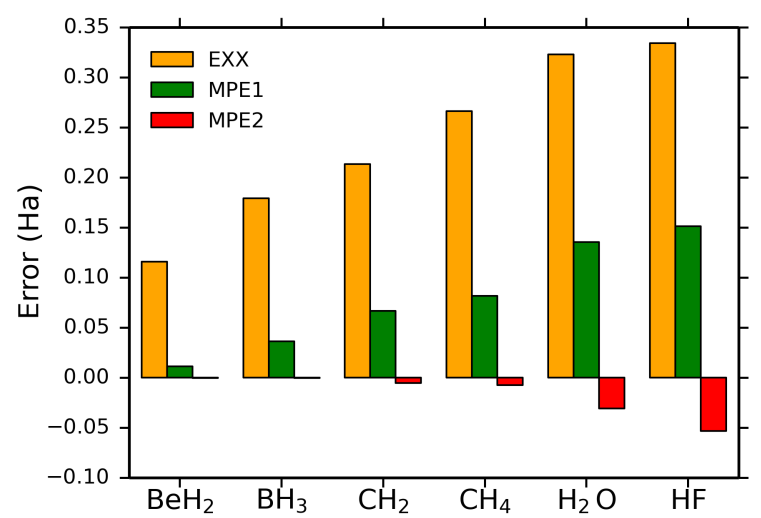

(b)

Figure 2: Errors of MPE energies for a series of small molecules using $\operatorname{CCSD}(\mathrm{T})$ results as the reference. (a) uDZ basis set. (b) uTZ basis set.

We apply MPE to a series of small molecules on their equilibrium geometries, including $\mathrm{BeH}_{2}, \mathrm{BH}_{3}, \mathrm{CH}_{2}, \mathrm{CH}_{4}, \mathrm{H}_{2} \mathrm{O}$ and $\mathrm{HF}$, and show the errors of total molecular energies in Figure 2. As can be seen, MPE at the first order (MPE1) reduces the errors of EXX significantly - more than $50 \%$ for all tested molecules in both basis sets, meaning that the correlation energy inside each electron pair is very large. However, MPE1 results still possess considerable errors, indicating that correcting the energy errors at only one-pair level is not enough. MPE at the second order (MPE2) further reduces the errors and gives very accurate total energies for $\mathrm{BeH}_{2}, \mathrm{BH}_{3}, \mathrm{CH}_{2}$ and $\mathrm{CH}_{4}$. MPE2 causes over correlations for $\mathrm{H}_{2} \mathrm{O}$ and HF, which may partly result from the low order of MPE expansion and numerical instability in the potential inversions. Overall, this result suggests that adding in correct pair-pair interactions is crucial for the accurate description of dynamic correlations in molecules. More importantly, Figure 2 demonstrates that when we apply successive higher order corrections, the MPE total energies become more accurate and converge quickly towards the highly accurate results. 
Comparing Figure 2a and Figure 2b, one may notice that the MPE results are more accurate in the larger uTZ basis set despite the larger EXX errors. As shown in Eq. 24 and Eq. 29, the quality of density matching in the potential inversions are determined by the size of the auxiliary basis set $\left\{\chi_{P}(\mathbf{r})\right\}$. Therefore, the superior performance of MPE in the larger basis set is probably due to the better density matching in the potential inversions, which implies MPE could be even more accurate when approaching the complete basis set limit.

In addition to the accuracy, we comment on the computational efficiency of MPE. Take $\mathrm{BH}_{3}$ in the uTZ basis set as an example. The computational time for each MPE1 correction for $\mathrm{BH}_{3}$ is around $1.5 \mathrm{~h}$ on $8 \mathrm{CPU}$ cores, while for each MPE2 correction the computational time increases to around $34 \mathrm{~h}$. As a comparison, the normal EXX calculation on $\mathrm{BH}_{3}$ only takes less than 1 min. Although it seems MPE corrections cost much more time than the DFT calculation, several points need to be emphasized. First, our high level calculation is full CI, which is known to be very expensive. If we switch the high level wavefunction theory to coupled cluster or truncated CI, the MPE computational efficiency will be improved significantly. Second, our code is still far away from optimized and many aspects need to be improved. For example, the interacting inversion routine uses finite differences to compute the gradient, which takes most of the computational time. Meanwhile, as a fragmentation method, MPE can be very well parallelized, which also needs to be explored in the future.

We then investigate the performance of MPE for four chemical reactions, as shown in Figure 3. In both basis sets, MPE1 improves the description of reaction energies over EXX for all chemical reactions considered. In the uDZ basis set (Figure 3a), MPE2 performs better than MPE1 in the first three reactions but worse in the last one. Although MPE provides successive improvement for the first three reactions, the absolute error of MPE2 reaction energy can still be larger than $5 \mathrm{kcal} / \mathrm{mol}$. However, in the larger uTZ basis set (Figure $3 \mathrm{~b}$ ), the MPE results get much better. There is clearly systematic improvement of MPE reaction energies for all four chemical reactions. Meanwhile, the absolute errors of MPE2 reaction energies are small in the uTZ basis set, all of which are below $2 \mathrm{kcal} / \mathrm{mol}$. This result again 


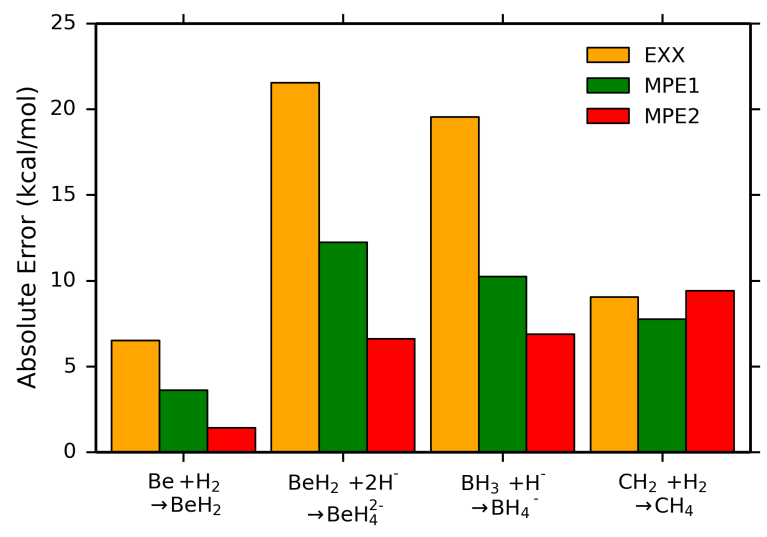

(a)

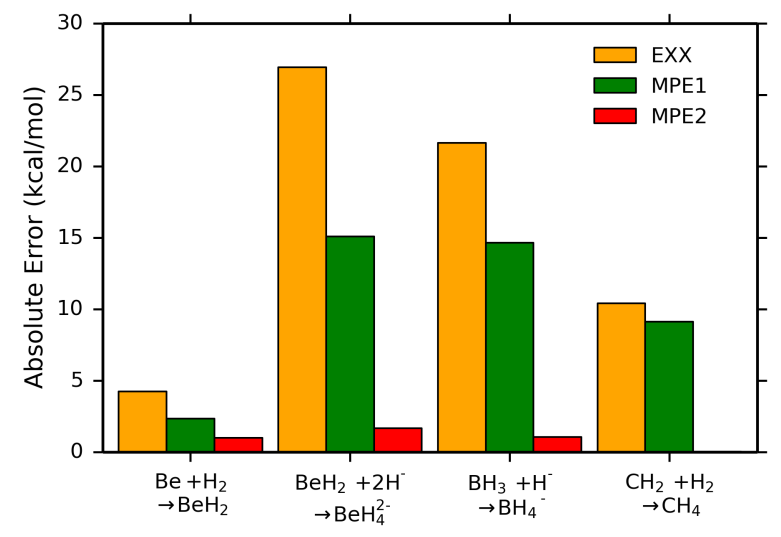

(b)

Figure 3: Absolute errors of MPE reaction energies using $\operatorname{CCSD}(\mathrm{T})$ results as the reference. (a) $\mathrm{uDZ}$ basis set. (b) uTZ basis set. Note that the MPE2 error for the last reaction in uTZ basis is not visible because it is very close to $0 \mathrm{kcal} / \mathrm{mol}$.

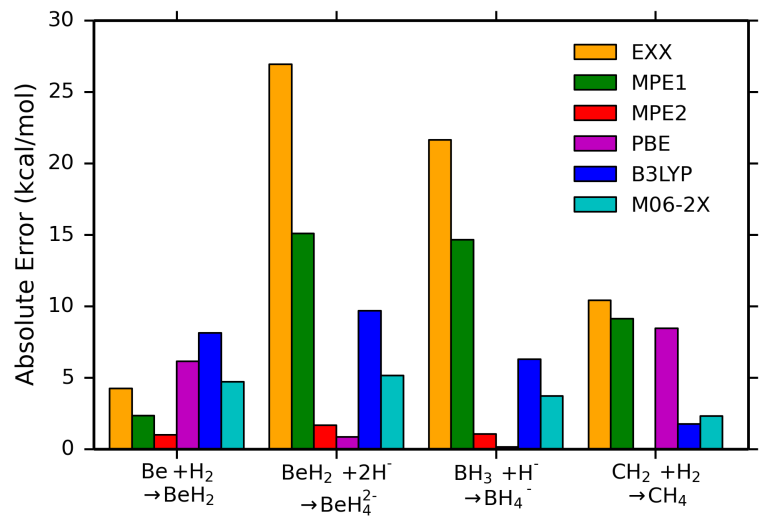

Figure 4: Absolute errors of MPE reaction energies compared to several commonly-used density functionals in the uTZ basis set. $\operatorname{CCSD}(\mathrm{T})$ results are used as the reference. Note that all MPE calculations use EXX as the starting functional.

shows the importance of accurate density matching in the potential inversions - the larger basis set results in better performance of MPE.

In Figure 4, we further compare the performance of MPE with several popular density functionals on the same four reactions in the uTZ basis set. The considered functionals involve PBE (GGA), ${ }^{95}$ B3LYP (hybrid GGA) ${ }^{17}$ and M06-2X (hybrid meta-GGA). ${ }^{92}$ One may notice that none of these three functionals is able to predict all reaction energies within $2 \mathrm{kcal} / \mathrm{mol}$ of error like MPE2. For example, PBE is the best functional for describing the 
second and third reactions, but it poses absolute errors of more than $5 \mathrm{kcal} / \mathrm{mol}$ for the other two reactions. Overall, Figures 3-4 demonstrate that, even with the worst performer EXX functional as the starting point, MPE is able to systematically correct the approximate functional and provides an accurate description of thermochemistry for tested chemical reactions at a low second order of expansion. Of course more extensive testing will be needed to establish whether these trends hold true for a wide variety of reactions, but the initial results are encouraging.

The thermochemistry of hydrogen molecular chains presents an interesting challenge for density functional approximations. In spite of the simple model, commonly-used GGA and hybrid functionals have been shown to fail in accurately describing the decomposition of hydrogen chains into hydrogen molecules. ${ }^{96,97}$ The failure is attributed to the inaccurate characterization of the delocalized electron distribution across more than one $\mathrm{H}_{2}$ unit, which is caused by the self-interaction error (delocalization error) present in these approximate functionals. We therefore test the performance of MPE on the decomposition of a hydrogen chain $\mathrm{H}_{2 \mathrm{n}}$ into $n$ hydrogen molecules, whose reaction energy can be calculated as:

$$
\Delta E=n E\left(H_{2}\right)-E\left(H_{2 n}\right),
$$

where $n$ is the number of $\mathrm{H}_{2}$ units in the hydrogen chain. The bond lengths between two adjacent $\mathrm{H}$ atoms in the hydrogen chains and hydrogen molecules are set to be $0.75 \AA$. The static correlation becomes another source of error for the tested methods due to the equal spacing between $\mathrm{H}$ atoms in the $\mathrm{H}_{2 \mathrm{n}}$ chain, but it is not dominant because of the small bond length.

The errors of the hydrogen chain decomposition energies per $\mathrm{H}_{2}$ unit compared to CCSD(T) is shown in Figure 5. The popular GGA functional PBE is shown to overestimate reaction energies severely: about $7 \mathrm{kcal} / \mathrm{mol}$ of error per $\mathrm{H}_{2}$ unit for $\mathrm{H}_{10}$ chain decomposition, indicating the large delocalization error in the PBE functional. On the other hand, the EXX 


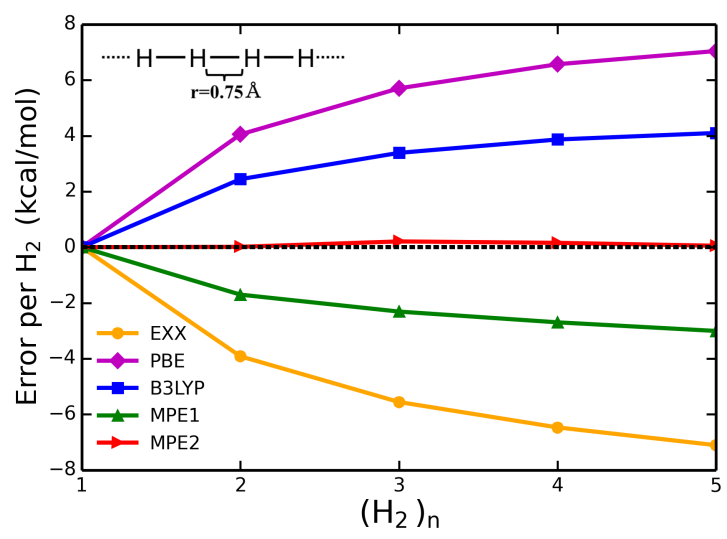

Figure 5: Errors of the hydrogen chain reaction energies per $\mathrm{H}_{2}$ unit in the $\mathrm{uDZ}$ basis set. $\mathrm{CCSD}(\mathrm{T})$ results are used as the reference.

functional significantly underestimates the reaction energies, with the similar magnitude of errors as PBE. This behavior is associated with the so-called localization error in literature, due to the neglect of electron correlation in the EXX functional. The hybrid functional B3LYP reduces the errors by one third compared to PBE because of the mixing of EXX, but still poses substantial errors. Furthermore, the errors of all three functionals increase as the hydrogen chain length becomes longer, which suggests the delocalization/localization errors are more severe in more extensive electron density distributions.

As seen in Figure 5, MPE1 provides a significant improvement over EXX, where the errors of reaction energies are already smaller than B3LYP by including the correct correlation for each electron pair in the hydrogen chain. The higher order MPE2 produces even better results: errors for different lengths of hydrogen chains are all reduced to almost zero, which indicates that including one-pair and pair-pair electron correlations is sufficient to remove all localization errors present in the EXX functional for hydrogen chains. In addition, this systematic improvement does not deteriorate as the chain length becomes longer, showing the ability of MPE to deal with systems with different electron density distributions. 


\subsection{Strong Correlation}

To test the performance of MPE on strongly correlated molecular systems, we study the symmetric bond breaking process in hydrogen rings using MPE. Hydrogen rings have re-

cently emerged as a benchmark for new electronic structure methods, ${ }^{98-101}$ as the strength of correlation can be tuned from weak to strong by changing the hydrogen atom spacing. Despite the simple model, the strong electron correlations present in stretched hydrogen rings require the correlation methods to be capable of treating full Coulomb interactions. Meanwhile, hydrogen rings in a realistic basis set have multiple orbitals per site as well as long-range interactions, making them more complicated compared to the one-dimensional (1D) Hubbard model. ${ }^{36}$ We have shown that MPE can deal with strong correlations in 1D Hubbard model very well, ${ }^{35}$ so it is interesting to investigate its performance on hydrogen rings, which reflects the challenge of real molecular systems.

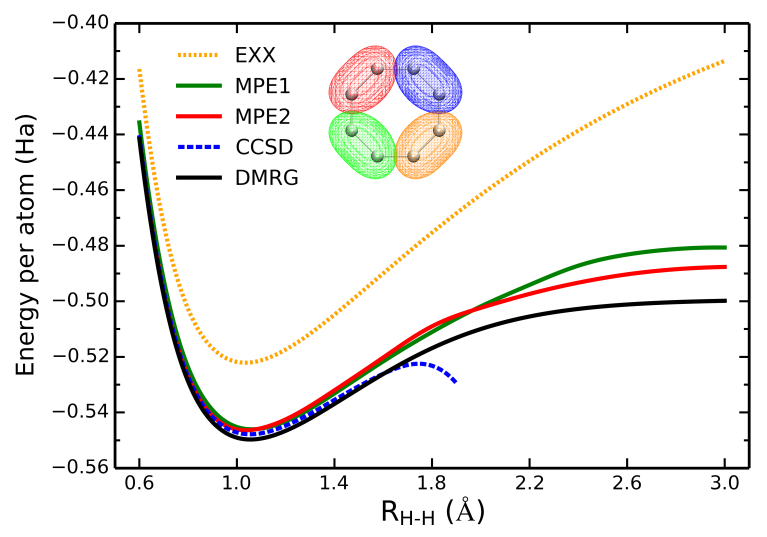

Figure 6: Energies per $\mathrm{H}$ atom in the $\mathrm{H}_{8}$ ring in the $\mathrm{uDZ}$ basis set. $\mathrm{SAH}$ decomposed pair densities are shown as an inset.

We first show the energies per $\mathrm{H}$ atom in the $\mathrm{H}_{8}$ ring in the $\mathrm{uDZ}$ basis set in Figure 6 . The DMRG result is presented here as a highly accurate answer for comparison. The EXX functional is shown to predict much higher energies compared to DMRG around the equilibrium distance, indicating it is incapable of describing dynamic correlations in the $\mathrm{H}_{8}$ ring. When the $\mathrm{H}-\mathrm{H}$ distance gets longer, the strong (static) correlation becomes more dominant, leading to even worse performance of EXX functional. This is expected for all single-determinantal 
approximate KS-DFT methods. Adding correct one-pair correlation at the MPE1 level results in a great improvement over EXX. The equilibrium-geometry result of MPE1 is already accurate compared to DMRG. More importantly, in the bond-breaking region, MPE1 reduces the EXX errors substantially because the use of FCI brings in correct multi-determinantal interactions at the two-electron level. This correction cannot be achieved using traditional single-reference correlation methods such as CCSD. As can be seen in Figure 6, CCSD describes the dynamic correlations very well but collapses when the $\mathrm{H}-\mathrm{H}$ distance is long. ${ }^{99}$ Despite the much better accuracy than EXX, it is clear that MPE1 is still insufficient for accurate estimation of strong correlations since the error per atom is still as high as $0.02 \mathrm{Ha}$ at $R_{H-H}=3.0 \AA$.

Including pair-pair electron correlations leads to further improvement in MPE2 compared to MPE1. MPE2 is still accurate around the equilibrium distance and further reduces the MPE1 errors at longer $\mathrm{H}-\mathrm{H}$ distances. Nevertheless, unlike in thermochemistry cases, even MPE2 cannot treat strong correlations in the stretched $\mathrm{H}_{8}$ ring accurately. This result can be attributed to two reasons. First, the second order of expansion for MPE may not be enough for this problem. Higher order of expansions, where six or more electron correlations are correctly computed, may be needed. Second, a more important reason might be that we employ the HF density as the input for MPE calculations here. As already shown in Figure 6, EXX, which is the counterpart method of HF in DFT, is a very poor solution for the strong correlation problems. Using bad input electron density from HF may cause MPE to finally converge to the inaccurate result, i.e., it is possible in this case even MPE4 is not satisfactory. This can be understood as the density-driven error in density functional theory. ${ }^{94,102}$ Therefore, to achieve a correct description of strong correlations, we may want to have better input electron density. Such density may be obtained through multi-configurational SCF calculations, or more ideally, from the optimization of self-consistent MPE calculations. We will not discuss the optimization of input density here and leave it for the future work.

We also apply MPE to the $\mathrm{H}_{6}$ ring in the $\mathrm{uDZ}$ basis set to test its performance for 


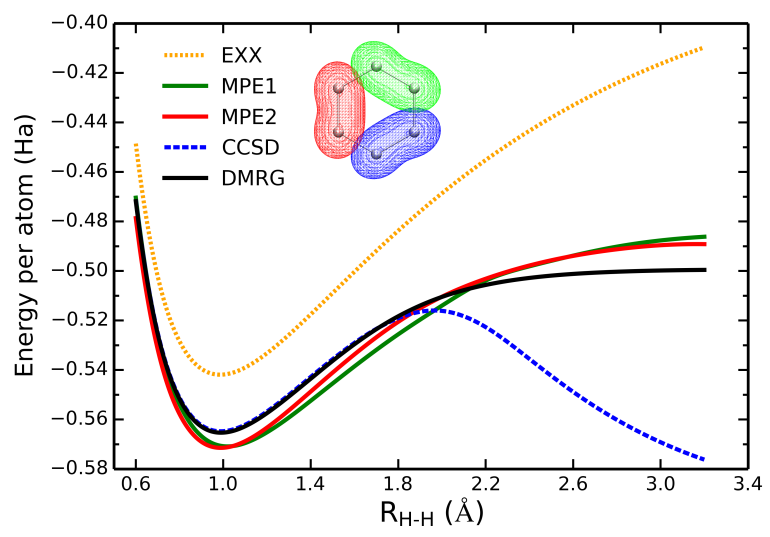

Figure 7: Energies per $\mathrm{H}$ atom in the $\mathrm{H}_{6}$ ring in the $\mathrm{uDZ}$ basis set. SAH decomposed pair densities are shown as an inset.

a hydrogen ring with different size, as demonstrated in Figure 7. MPE1 and MPE2 give similar accuracy at long H-H distances compared to Figure 7: MPE1 provides a significant improvement over EXX and MPE2 further reduces the errors of MPE1, while both MPE1 and MPE2 pose errors of more than $0.01 \mathrm{Ha}$ per atom at $R_{H-H}=3.2 \AA$. Around the equilibrium distance, however, the performance of MPE is not as satisfactory as in the $\mathrm{H}_{8}$ ring. Both MPE methods overcorrelate for about 0.01 Ha per atom compared to DMRG and MPE2 provides no improvement over MPE1. This result is surprising considering there are only six electrons in this system, meaning that MPE3 is the "exact" answer. We suspect such behavior is related to the aromaticity of the system: the $\mathrm{H}_{6}$ ring can be viewed as an aromatic ring while the $\mathrm{H}_{8}$ ring is anti-aromatic. In aromatic systems, specific order of MPE (for example, MPE3) and better density decomposition may be needed to achieve high accuracy, which is also found in a previous MPE study of benzene dimer in the PPP model. ${ }^{40}$

\subsection{Dependence on Localization Parameter $\alpha$}

All MPE results shown above are based on pair densities generated by the SAH decomposition using the localization parameter $\alpha=4$ for Eq. 20. We choose $\alpha=4$ because it is the maximum value that still maintains smoothness in pair densities so that the ill-conditioning problems in potential inversions can be mostly avoided. Meanwhile, the resulting pair densi- 
ties are localized enough to make sure MPE energies converge quickly to the accurate result. Here, we test the performance of MPE with different $\alpha$ values $(\alpha=1.5,2,3,4)$ to show its dependence on the parameter $\alpha$.

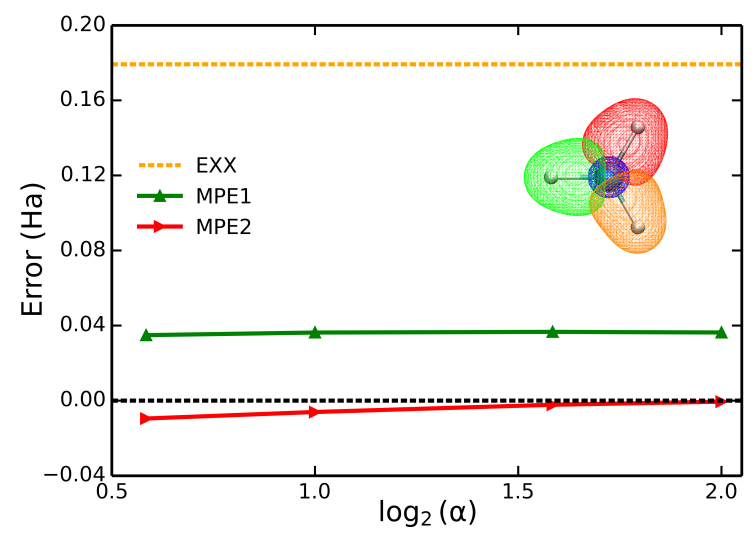

(a)

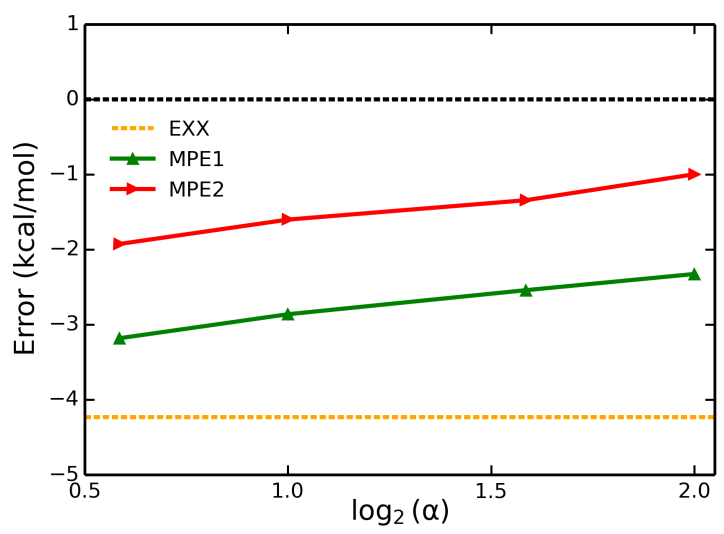

(b)

Figure 8: The dependence of MPE energies on the localization parameter $\alpha$ in the uTZ basis set. $\operatorname{CCSD}(\mathrm{T})$ results are used as the reference. (a) Total energy of $\mathrm{BH}_{3}$. (b) Reaction energy of $\mathrm{Be}+\mathrm{H}_{2} \rightarrow \mathrm{BeH}_{2}$.

We fist show the dependence of MPE on the localization parameter $\alpha$ for the total molecular energy of $\mathrm{BH}_{3}$ in Figure 8a. For all four $\alpha$ values tested, the MPE1 energy is quite stable, while the MPE2 energy becomes slightly better when larger $\alpha$ value is used. Similar results can be observed in Figure 8b, where we test the dependence of MPE on $\alpha$ for the reaction energy of $\mathrm{Be}+\mathrm{H}_{2} \rightarrow \mathrm{BeH}_{2}$. Both MPE1 and MPE2 predict better reaction energies when larger $\alpha$ values are used. However, the difference of MPE2 reaction energy for using $\alpha=1.5$ and $\alpha=4$ is just $1 \mathrm{kcal} / \mathrm{mol}$. Overall speaking, we find that more localized SAH decomposition (larger $\alpha$ value) leads to better accuracy of MPE, and the dependence of MPE on the parameter $\alpha$ is weak. Thus, we believe $\alpha=4$ is a suitable choice for this study, and $\alpha \geq 4$ should be used in the future MPE study as long as the potential inversions remain numerically stable. 


\section{Conclusions}

In this work, we extend the many-pair expansion to molecular systems with ab initio Hamiltonians. We employ the self-attractive Hartree decomposition to generate localized and $v$-representable pair densities as the basic fragment densities in molecular MPE. To perform numerically stable potential inversions, we introduce auxiliary basis sets to represent the external potentials and construct the orbital and potential basis sets in a balanced fashion. Our numerical study shows that MPE predicts accurate molecular and reaction energies for a few small molecules and hydrogen chains, even with the low-level EXX functional and a low second order of expansion. The description of thermochemistry for tested chemical reactions is systematically improved as successive higher order MPE corrections are applied. Meanwhile, through the hydrogen chain case, we demonstrate the potential of MPE to serve as a method to systemically remove the many-electron self-interaction error in approximate density functionals. The study of bond breaking processes in hydrogen rings exhibits the ability of MPE to deal with strong correlations, but also points out the need for better input density or density decomposition in MPE. In conclusion, this work shows that MPE provides a promising framework to systematically improve density functional calculations of molecules.

The future work of MPE should focus on four aspects. First, more realistic density functionals should be used to test the performance of MPE. In addition to EXX, we should test whether MPE can also systematically improve the DFT calculations with commonly-used LDA, GGA, meta-GGA and hybrid functionals. Especially, we want to investigate how MPE can be applied to remove the delocalization error and strong correlation error in these functionals. Second, faster implementation is needed for applying MPE to more realistic

chemical systems. Although the MPEm correction is an $O\left(N^{m}\right)$ method $(N$ is the number of electron pairs), its speed is hindered by the large prefactor due to expensive interacting potential inversion calculations. To make MPE faster, two different directions may be explored. (a) Simplify the potential inversion scheme in MPE. One possible solution is to 
combine DFT embedding methods ${ }^{103,104}$ with MPE, to turn MPE into a many potential expansion problem. Instead of partitioning the density, we can partition the external potential into small fragments. In this way, the most expensive interacting potential inversions can be avoided. Another possible way is to apply the projection embedding scheme ${ }^{105}$ to avoid all potential inversions. We also note that the method of Staroverov ${ }^{106}$ to obtain KS potentials from interacting wavefunctions could potentially solve some of MPE's unstable potential inversion problems, especially when the potential and orbital basis sets are not balanced. (b) Exploit the locality in SAH fragment densities and use local correlation methods ${ }^{107-109}$ as the alternative to FCI. Third, a density optimization scheme is needed for more accurate MPE calculations. MPE is formally a density functional hierarchy that converges to the exact energy. However, in reality, MPE results may converge to the inaccurate answer due to the use of approximate DFT density. Therefore, a self-consistent MPE scheme may be needed to optimize the input density and correct the density errors. Fourth, MPE may be used as a tool to develop better density functionals. As shown in this work, MPE is able to reveal how approximate functionals perform for particular fragment densities by comparing with the exact correlation and interaction energies from FCI. New density functionals can thus be designed by optimally tuning parameters (e.g., range-separation parameter, percentage of EXX) for specific molecule so that certain fragment correction/interaction energies (e.g., pair-pair interactions) can be accurately described, leading to improved description of the whole molecule.

\section{Acknowledgement}

This work was funded by a grant from the NSF (CHE-1464804). T.V. acknowledges support from a David and Lucile Packard Foundation Fellowship. T.Z. acknowledges support from the MIT Lester Wolfe Fellowship. 


\section{Supporting Information Available}

The detailed description of constructing auxiliary basis sets and the energy errors for non$v$-representable densities.

\section{References}

(1) Kohn, W. Nobel Lecture: Electronic structure of matter-wave functions and density functionals. Rev. Mod. Phys. 1999, 71, 1253.

(2) Burke, K. Perspective on density functional theory. J. Chem. Phys. 2012, 136, 150901.

(3) Becke, A. D. Perspective: Fifty years of density-functional theory in chemical physics. J. Chem. Phys. 2014, 140, 18A301.

(4) Hohenberg, P.; Kohn, W. Inhomogeneous electron gas. Phys. Rev. 1964, 136, B864.

(5) Kohn, W.; Sham, L. J. Self-consistent equations including exchange and correlation effects. Phys. Rev. 1965, 140, A1133.

(6) Cohen, A. J.; Mori-Sánchez, P.; Yang, W. Challenges for density functional theory. Chem. Rev. 2011, 112, 289-320.

(7) Perdew, J. P.; Zunger, A. Self-interaction correction to density-functional approximations for many-electron systems. Phys. Rev. B 1981, 23, 5048.

(8) Ruzsinszky, A.; Perdew, J. P.; Csonka, G. I.; Vydrov, O. A.; Scuseria, G. E. Spurious fractional charge on dissociated atoms: Pervasive and resilient self-interaction error of common density functionals. J. Chem. Phys. 2006, 125, 194112.

(9) Mori-Sánchez, P.; Cohen, A. J.; Yang, W. Localization and delocalization errors in density functional theory and implications for band-gap prediction. Phys. Rev. Lett. 2008, 100, 146401. 
(10) Dreuw, A.; Head-Gordon, M. Single-reference ab initio methods for the calculation of excited states of large molecules. Chem. Rev. 2005, 105, 4009-4037.

(11) Hait, D.; Zhu, T.; McMahon, D. P.; Van Voorhis, T. Prediction of Excited-State Energies and Singlet-Triplet Gaps of Charge-Transfer States Using a Restricted OpenShell Kohn-Sham Approach. J. Chem. Theory Comput. 2016, 12, 3353-3359.

(12) Cohen, A. J.; Mori-Sánchez, P.; Yang, W. Fractional spins and static correlation error in density functional theory. J.Chem. Phys. 2008, 129, 121104.

(13) Cohen, A. J.; Mori-Sánchez, P.; Yang, W. Insights into current limitations of density functional theory. Science 2008, 321, 792-794.

(14) Sherrill, C. D. Frontiers in electronic structure theory. J. Chem. Phys. 2010, 132, 110902.

(15) Kristyán, S.; Pulay, P. Can (semi) local density functional theory account for the London dispersion forces? Chem. Phys. Lett. 1994, 229, 175-180.

(16) Allen, M. J.; Tozer, D. J. Helium dimer dispersion forces and correlation potentials in density functional theory. J. Chem. Phys. 2002, 117, 11113-11120.

(17) Becke, A. D. Density-functional thermochemistry. III. The role of exact exchange. J. Chem. Phys. 1993, 98, 5648-5652.

(18) Vydrov, O. A.; Scuseria, G. E. Assessment of a long-range corrected hybrid functional. J. Chem. Phys. 2006, 125, 234109.

(19) Vydrov, O. A.; Scuseria, G. E. Effect of the Perdew-Zunger self-interaction correction on the thermochemical performance of approximate density functionals. J. Chem. Phys. 2004, 121, 8187-8193. 
(20) Li, C.; Zheng, X.; Su, N. Q.; Yang, W. Localized Orbital Scaling Correction for Systematic Elimination of Delocalization Error in Density Functional Approximations. Natl. Sci. Rev. 2017,

(21) Anisimov, V. I.; Zaanen, J.; Andersen, O. K. Band theory and Mott insulators: Hubbard U instead of Stoner I. Phys. Rev. B 1991, 44, 943.

(22) Stoudenmire, E.; Wagner, L. O.; White, S. R.; Burke, K. One-dimensional continuum electronic structure with the density-matrix renormalization group and its implications for density-functional theory. Phys. Rev. Lett. 2012, 109, 056402.

(23) Li Manni, G.; Carlson, R. K.; Luo, S.; Ma, D.; Olsen, J.; Truhlar, D. G.; Gagliardi, L. Multiconfiguration pair-density functional theory. J. Chem. Theory Comput. 2014, 10, 3669-3680.

(24) Malet, F.; Gori-Giorgi, P. Strong correlation in Kohn-Sham density functional theory. Phys. Rev. Lett. 2012, 109, 246402.

(25) Wu, Q.; Yang, W. Empirical correction to density functional theory for van der Waals interactions. J. Chem. Phys. 2002, 116, 515-524.

(26) Grimme, S.; Antony, J.; Ehrlich, S.; Krieg, H. A consistent and accurate ab initio parametrization of density functional dispersion correction (DFT-D) for the 94 elements H-Pu. J. Chem. Phys. 2010, 132, 154104.

(27) Becke, A. D.; Johnson, E. R. A density-functional model of the dispersion interaction. J. Chem. Phys. 2005, 123, 154101.

(28) Tkatchenko, A.; DiStasio Jr, R. A.; Car, R.; Scheffler, M. Accurate and efficient method for many-body van der Waals interactions. Phys. Rev. Lett. 2012, 108, 236402.

(29) Vydrov, O. A.; Van Voorhis, T. Nonlocal van der Waals density functional made simple. Phys. Rev. Lett. 2009, 103, 063004. 
(30) Vydrov, O. A.; Van Voorhis, T. Nonlocal van der Waals density functional: The simpler the better. J. Chem. Phys. 2010, 133, 244103.

(31) Lee, K.; Murray, É. D.; Kong, L.; Lundqvist, B. I.; Langreth, D. C. Higher-accuracy van der Waals density functional. Phys. Rev. B 2010, 82, 081101.

(32) Schwabe, T.; Grimme, S. Double-hybrid density functionals with long-range dispersion corrections: higher accuracy and extended applicability. Phys. Chem. Chem. Phys. 2007, 9, 3397-3406.

(33) Furche, F.; Van Voorhis, T. Fluctuation-dissipation theorem density-functional theory. J. Chem. Phys. 2005, 122, 164106.

(34) Perdew, J. P.; Ruzsinszky, A.; Tao, J.; Staroverov, V. N.; Scuseria, G. E.; Csonka, G. I. Prescription for the design and selection of density functional approximations: More constraint satisfaction with fewer fits. J. Chem. Phys. 2005, 123, 062201.

(35) Zhu, T.; de Silva, P.; van Aggelen, H.; Van Voorhis, T. Many-electron expansion: A density functional hierarchy for strongly correlated systems. Phys. Rev. B 2016, 93, 201108.

(36) Hubbard, J. Electron correlations in narrow energy bands. Proceedings of the Royal Society of London A: Mathematical, Physical and Engineering Sciences. 1963; pp $238-257$.

(37) Peierls, R. E. Quantum theory of solids; Oxford University Press, 1955.

(38) Pariser, R.; Parr, R. G. A Semi-Empirical Theory of the Electronic Spectra and Electronic Structure of Complex Unsaturated Molecules. I. J. Chem. Phys. 1953, 21, $466-471$.

(39) Pople, J. Electron interaction in unsaturated hydrocarbons. Trans. Faraday Soc. 1953, 49, 1375-1385. 
(40) de Silva, P.; Zhu, T.; Van Voorhis, T. Long-range interactions from the many-pair expansion: A different avenue to dispersion in DFT. J. Chem. Phys. 2017, 146, 024111.

(41) Zhu, T.; de Silva, P.; Van Voorhis, T. Self-Attractive Hartree Decomposition: Partitioning Electron Density into Smooth Localized Fragments. J. Chem. Theory Comput. 2018, 14, 92-103.

(42) Lieb, E. H. Inequalities; Springer, 2002; pp 269-303.

(43) Bendazzoli, G. L.; Evangelisti, S. Computation and analysis of the full configuration interaction wave function of some simple systems. Int. J. of Quant. Chem. 1993, 48, 287-301.

(44) Bendazzoli, G. L.; Evangelisti, S. A vector and parallel full configuration interaction algorithm. J. Chem. Phys. 1993, 98, 3141-3150.

(45) Lieb, E. H. Density functionals for coulomb systems. Int. J. Quantum Chem. 1983, 24, 243-277.

(46) Colonna, F.; Savin, A. Correlation energies for some two-and four-electron systems along the adiabatic connection in density functional theory. J. Chem. Phys. 1999, 110, 2828-2835.

(47) Teale, A.; Coriani, S.; Helgaker, T. The calculation of adiabatic-connection curves from full configuration-interaction densities: Two-electron systems. J. Chem. Phys. 2009, 130, 104111.

(48) Kümmel, S.; Kronik, L. Orbital-dependent density functionals: Theory and applications. Rev. Mod. Phys. 2008, 80, 3.

(49) Cui, J.; Liu, H.; Jordan, K. D. Theoretical Characterization of the (H2O) 21 Cluster: Application of an n-body Decomposition Procedure. J. Phys. Chem. B 2006, 110, $18872-18878$. 
(50) Richard, R. M.; Herbert, J. M. A generalized many-body expansion and a unified view of fragment-based methods in electronic structure theory. J. Chem. Phys. 2012, 137, 064113.

(51) Stoll, H. On the correlation energy of graphite. J. Chem. Phys. 1992, 97, 8449-8454.

(52) Hedin, L. New method for calculating the one-particle Green's function with application to the electron-gas problem. Phys. Rev. 1965, 139, A796.

(53) Č́žzek, J. On the correlation problem in atomic and molecular systems. Calculation of wavefunction components in Ursell-type expansion using quantum-field theoretical methods. J. Chem. Phys. 1966, 45, 4256-4266.

(54) Zhao, Q.; Morrison, R. C.; Parr, R. G. From electron densities to Kohn-Sham kinetic energies, orbital energies, exchange-correlation potentials, and exchange-correlation energies. Phys. Rev. A 1994, 50, 2138.

(55) Wu, Q.; Yang, W. A direct optimization method for calculating density functionals and exchange-correlation potentials from electron densities. J. Chem. Phys. 2003, $118,2498-2509$.

(56) Van Leeuwen, R.; Baerends, E. Exchange-correlation potential with correct asymptotic behavior. Phys. Rev. A 1994, 49, 2421.

(57) Press, W. H. Numerical recipes 3rd edition: The art of scientific computing; Cambridge university press, 2007.

(58) Wagner, L. O.; Baker, T. E.; Stoudenmire, E.; Burke, K.; White, S. R. Kohn-Sham calculations with the exact functional. Phys. Rev. B 2014, 90, 045109.

(59) Kohn, W. v-Representability and density functional theory. Phys. Rev. Lett. 1983, 51, 1596. 
(60) Savin, A.; Wesolowski, T. A. Advances in the Theory of Atomic and Molecular Systems; Springer, 2009; pp 311-326.

(61) de Silva, P.; Wesolowski, T. A. Exact non-additive kinetic potentials in realistic chemical systems. J. Chem. Phys. 2012, 13\%, 094110.

(62) de Silva, P.; Wesolowski, T. A. Pure-state noninteracting v-representability of electron densities from Kohn-Sham calculations with finite basis sets. Phys. Rev. A 2012, 85, 032518.

(63) Mori-Sánchez, P.; Cohen, A. J.; Yang, W. Many-electron self-interaction error in approximate density functionals. J. Chem. Phys. 2006, 125, 201102.

(64) Ruzsinszky, A.; Perdew, J. P.; Csonka, G. I.; Vydrov, O. A.; Scuseria, G. E. Density functionals that are one-and two-are not always many-electron self-interaction-free, as shown for H. J. Chem. Phys. 2007, 126, 104102.

(65) Vydrov, O. A.; Scuseria, G. E.; Perdew, J. P.; Ruzsinszky, A.; Csonka, G. I. Scaling down the Perdew-Zunger self-interaction correction in many-electron regions. J. Chem. Phys. 2006, 124, 094108.

(66) Boys, S. F. Construction of some molecular orbitals to be approximately invariant for changes from one molecule to another. Rev. Mod. Phys. 1960, 32, 296.

(67) Paulus, B. The method of incrementsâĂ $\breve{T} a$ wavefunction-based ab initio correlation method for solids. Phys. Rep. 2006, 428, 1-52.

(68) Edmiston, C.; Ruedenberg, K. Localized atomic and molecular orbitals. Rev. Mod. Phys. 1963, 35, 457.

(69) Hartree, D. R. The calculation of atomic structures; Wiley, 1957.

(70) Whitten, J. L. Coulombic potential energy integrals and approximations. J. Chem. Phys. 1973, 58, 4496-4501. 
(71) Weigend, F.; Köhn, A.; Hättig, C. Efficient use of the correlation consistent basis sets in resolution of the identity MP2 calculations. J. Chem. Phys. 2002, 116, 3175-3183.

(72) Görling, A. New KS method for molecules based on an exchange charge density generating the exact local KS exchange potential. Phys. Rev. Lett. 1999, 83, 5459.

(73) Dunlap, B. I.; Connolly, J. W.; Sabin, J. R. On the applicability of LCAO-X $\alpha$ methods to molecules containing transition metal atoms: The nickel atom and nickel hydride. Int. J. Quantum Chem. 1977, 12, 81-87.

(74) Dunlap, B. I.; Connolly, J.; Sabin, J. On some approximations in applications of X $\alpha$ theory. J. Chem. Phys. 1979, 71, 3396-3402.

(75) Wu, Q.; Yang, W. Algebraic equation and iterative optimization for the optimized effective potential in density functional theory. J. Theor. Comput. Chem. 2003, 2, $627-638$.

(76) Purvis III, G. D.; Bartlett, R. J. A full coupled-cluster singles and doubles model: The inclusion of disconnected triples. J. Chem. Phys. 1982, 76, 1910-1918.

(77) Dunning Jr, T. H. Gaussian basis sets for use in correlated molecular calculations. I. The atoms boron through neon and hydrogen. J. Chem. Phys. 1989, 90, 1007-1023.

(78) Shao, Y.; Gan, Z.; Epifanovsky, E.; Gilbert, A. T. B.; Wormit, M.; Kussmann, J.; Lange, A. W.; Behn, A.; Deng, J.; Feng, X.; Ghosh, D.; Goldey, M.; Horn, P. R.; Jacobson, L. D.; Kaliman, I.; Khaliullin, R. Z.; Kús, T.; Landau, A.; Liu, J.; Proynov, E. I.; Rhee, Y. M.; Richard, R. M.; Rohrdanz, M. A.; Steele, R. P.; Sundstrom, E. J.; Woodcock III, H. L.; Zimmerman, P. M.; Zuev, D.; Albrecht, B.; Alguire, E.; Austin, B.; Beran, G. J. O.; Bernard, Y. A.; Berquist, E.; Brandhorst, K.; Bravaya, K. B.; Brown, S. T.; Casanova, D.; Chang, C.-M.; Chen, Y.; Chien, S. H.; Closser, K. D.; Crittenden, D. L.; Diedenhofen, M.; DiStasio Jr., R. A.; Dop, H.; Dutoi, A. D.; 
Edgar, R. G.; Fatehi, S.; Fusti-Molnar, L.; Ghysels, A.; Golubeva-Zadorozhnaya, A.; Gomes, J.; Hanson-Heine, M. W. D.; Harbach, P. H. P.; Hauser, A. W.; Hohenstein, E. G.; Holden, Z. C.; Jagau, T.-C.; Ji, H.; Kaduk, B.; Khistyaev, K.; Kim, J.; Kim, J.; King, R. A.; Klunzinger, P.; Kosenkov, D.; Kowalczyk, T.; Krauter, C. M.; Lao, K. U.; Laurent, A.; Lawler, K. V.; Levchenko, S. V.; Lin, C. Y.; Liu, F.; Livshits, E.; Lochan, R. C.; Luenser, A.; Manohar, P.; Manzer, S. F.; Mao, S.-P.; Mardirossian, N.; Marenich, A. V.; Maurer, S. A.; Mayhall, N. J.; Oana, C. M.; Olivares-Amaya, R.; O’Neill, D. P.; Parkhill, J. A.; Perrine, T. M.; Peverati, R.; Pieniazek, P. A.; Prociuk, A.; Rehn, D. R.; Rosta, E.; Russ, N. J.; Sergueev, N.; Sharada, S. M.; Sharmaa, S.; Small, D. W.; Sodt, A.; Stein, T.; Stück, D.; Su, Y.-C.; Thom, A. J. W.; Tsuchimochi, T.; Vogt, L.; Vydrov, O.; Wang, T.; Watson, M. A.; Wenzel, J.; White, A.; Williams, C. F.; Vanovschi, V.; Yeganeh, S.; Yost, S. R.; You, Z.-Q.; Zhang, I. Y.; Zhang, X.; Zhou, Y.; Brooks, B. R.; Chan, G. K. L.; Chipman, D. M.; Cramer, C. J.; Goddard III, W. A.; Gordon, M. S.; Hehre, W. J.; Klamt, A.; Schaefer III, H. F.; Schmidt, M. W.; Sherrill, C. D.; Truhlar, D. G.; Warshel, A.; Xua, X.; Aspuru-Guzik, A.; Baer, R.; Bell, A. T.; Besley, N. A.; Chai, J.-D.; Dreuw, A.; Dunietz, B. D.; Furlani, T. R.; Gwaltney, S. R.; Hsu, C.P.; Jung, Y.; Kong, J.; Lambrecht, D. S.; Liang, W.; Ochsenfeld, C.; Rassolov, V. A.; Slipchenko, L. V.; Subotnik, J. E.; Van Voorhis, T.; Herbert, J. M.; Krylov, A. I.; Gill, P. M. W.; Head-Gordon, M. Advances in molecular quantum chemistry contained in the Q-Chem 4 program package. Mol. Phys. 2015, 113, 184-215.

(79) Raghavachari, K.; Trucks, G. W.; Pople, J. A.; Head-Gordon, M. A fifth-order perturbation comparison of electron correlation theories. Chem. Phys. Lett. 1989, 157, $479-483$.

(80) White, S. R. Density matrix formulation for quantum renormalization groups. Phys. Rev. Lett. 1992, 69, 2863. 
(81) Sun, Q.; Berkelbach, T. C.; Blunt, N. S.; Booth, G. H.; Guo, S.; Li, Z.; Liu, J.; McClain, J. D.; Sayfutyarova, E. R.; Sharma, S.; Wouters, S.; Chan, G. K.-L. PySCF: the Python-based simulations of chemistry framework. Wiley Interdiscip. Rev. Comput. Mol. Sci. 2018, 8 .

(82) Chan, G. K.-L.; Head-Gordon, M. Highly correlated calculations with a polynomial cost algorithm: A study of the density matrix renormalization group. J. Chem. Phys. 2002, 116, 4462-4476.

(83) Olivares-Amaya, R.; Hu, W.; Nakatani, N.; Sharma, S.; Yang, J.; Chan, G. K.-L. The ab-initio density matrix renormalization group in practice. J. Chem. Phys. 2015, 142, 034102.

(84) Staroverov, V. N.; Scuseria, G. E.; Davidson, E. R. Optimized effective potentials yielding Hartree-Fock energies and densities. J. Chem. Phys. 2006, 124, 141103.

(85) Hirata, S.; Ivanov, S.; Grabowski, I.; Bartlett, R. J.; Burke, K.; Talman, J. D. Can optimized effective potentials be determined uniquely? J. Chem. Phys. 2001, 115, $1635-1649$.

(86) Heßelmann, A.; Götz, A. W.; Della Sala, F.; Görling, A. Numerically stable optimized effective potential method with balanced Gaussian basis sets. J. Chem. Phys. 2007, 127, 054102.

(87) Bulat, F. A.; Heaton-Burgess, T.; Cohen, A. J.; Yang, W. Optimized effective potentials from electron densities in finite basis sets. J. Chem. Phys. 2007, 127, 174101.

(88) Jacob, C. R. Unambiguous optimization of effective potentials in finite basis sets. $J$. Chem. Phys. 2011, 135, 244102.

(89) Ryabinkin, I. G.; Kananenka, A. A.; Staroverov, V. N. Accurate and efficient approx- 
imation to the optimized effective potential for exchange. Phys. Rev. Lett. 2013, 111, 013001.

(90) Humphrey, W.; Dalke, A.; Schulten, K. VMD: visual molecular dynamics. J. Mol. Graph. 1996, 14, 33-38.

(91) Schaftenaar, G.; Noordik, J. H. Molden: a pre-and post-processing program for molecular and electronic structures. J. Comput. Aid. Mol. Des. 2000, 14, 123-134.

(92) Zhao, Y.; Truhlar, D. G. The M06 suite of density functionals for main group thermochemistry, thermochemical kinetics, noncovalent interactions, excited states, and transition elements: two new functionals and systematic testing of four M06-class functionals and 12 other functionals. Theor. Chem. Acc. 2008, 120, 215-241.

(93) Zhang, Y.; Xu, X.; Goddard, W. A. Doubly hybrid density functional for accurate descriptions of nonbond interactions, thermochemistry, and thermochemical kinetics. Proc. Natl. Acad. Sci. U.S.A. 2009, 106, 4963-4968.

(94) Kim, M.-C.; Sim, E.; Burke, K. Understanding and reducing errors in density functional calculations. Phys. Rev. Lett. 2013, 111, 073003.

(95) Perdew, J. P.; Burke, K.; Ernzerhof, M. Generalized gradient approximation made simple. Phys. Rev. Lett. 1996, 77, 3865.

(96) Zheng, X.; Liu, M.; Johnson, E. R.; Contreras-García, J.; Yang, W. Delocalization error of density-functional approximations: A distinct manifestation in hydrogen molecular chains. J. Chem. Phys. 2012, 137, 214106.

(97) Su, N. Q.; Xu, X. Error accumulations in adhesive energies of dihydrogen molecular chains: performances of the XYG3 type of doubly hybrid density functionals. J. Phys. Chem. A 2014, 119, 1590-1599. 
(98) Knizia, G.; Chan, G. K.-L. Density matrix embedding: A strong-coupling quantum embedding theory. J. Chem. Theory. Comput. 2013, 9, 1428-1432.

(99) Bulik, I. W.; Henderson, T. M.; Scuseria, G. E. Can single-reference coupled cluster theory describe static correlation? J. Chem. Theory. Comput. 2015, 11, 3171-3179.

(100) Pham, H. Q.; Bernales, V.; Gagliardi, L. Can Density Matrix Embedding Theory with the Complete Activate Space Self-Consistent Field Solver Describe Single and Double Bond Breaking in Molecular Systems? J. Chem. Theory Comput. 2018, 14, 1960-1968.

(101) Ye, H.-Z.; Welborn, M.; Ricke, N. D.; Van Voorhis, T. Incremental embedding: A density matrix embedding scheme for molecules. J. Chem. Phys. 2018, 149, 194108.

(102) Kim, M.-C.; Sim, E.; Burke, K. Ions in solution: Density corrected density functional theory (DC-DFT). J. Chem. Phys. 2014, 140, 18A528.

(103) Goodpaster, J. D.; Barnes, T. A.; Manby, F. R.; Miller III, T. F. Density functional theory embedding for correlated wavefunctions: Improved methods for open-shell systems and transition metal complexes. J. Chem. Phys. 2012, 137, 224113.

(104) Huang, C.; Pavone, M.; Carter, E. A. Quantum mechanical embedding theory based on a unique embedding potential. J. Chem. Phys. 2011, 134, 154110.

(105) Manby, F. R.; Stella, M.; Goodpaster, J. D.; Miller III, T. F. A simple, exact densityfunctional-theory embedding scheme. J. Chem. Theory Comput. 2012, 8, 2564-2568.

(106) Ryabinkin, I. G.; Kohut, S. V.; Staroverov, V. N. Reduction of electronic wave functions to Kohn-Sham effective potentials. Phys. Rev. Lett. 2015, 115, 083001.

(107) Schütz, M.; Werner, H.-J. Low-order scaling local electron correlation methods. IV. Linear scaling local coupled-cluster (LCCSD). J. Chem. Phys. 2001, 114, 661-681. 
(108) Riplinger, C.; Neese, F. An efficient and near linear scaling pair natural orbital based local coupled cluster method. J. Chem. Phys. 2013, 138, 034106.

(109) Yang, J.; Chan, G. K.-L.; Manby, F. R.; Schütz, M.; Werner, H.-J. The orbital-specificvirtual local coupled cluster singles and doubles method. J. Chem. Phys. 2012, 136, 144105. 


\section{Graphical TOC Entry}

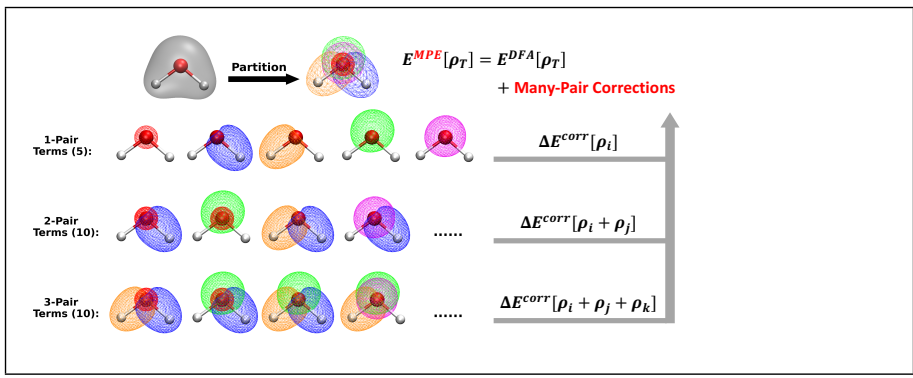

